Check for updates

Cite this: Chem. Commun., 2020, 56, 12272

Received 24th June 2020

Accepted 9th September 2020

DOI: $10.1039 / \mathrm{d} 0 \mathrm{cc} 04387 \mathrm{~g}$

rsc.li/chemcomm

\section{Photoelectrocatalytic production of solar fuels with semiconductor oxides: materials, activity and modeling}

\author{
Damián Monllor-Satoca, (D) María Isabel Díez-García, (D) † Teresa Lana-Villarreal (iD \\ and Roberto Gómez (iD *
}

\begin{abstract}
Oxide photoelectrochemistry has been under continuous development over the last half century. These decades have witnessed the use of electrodes of different nature (from single crystals to nanoparticulate films), new electrode materials (including ternary and multinary transition metal oxides), and different strategies for improving their efficiency and stability (e.g. doping or protective layers). Although the very high initial expectations for using oxide electrodes in solar energy conversion were not fully met, substantial efforts have been devoted to reach an in-depth understanding of the processes limiting their functioning, providing firm bases for further developments. In this article, we review our main contributions in this field; in particular, we focus on the water photooxidation (i.e. oxygen evolution reaction), water photoreduction (i.e. hydrogen evolution reaction) and full water splitting processes (in a tandem cell) with binary and ternary oxides, including metal hydroxides as co-catalysts. We emphasize the importance of modeling and obtaining mechanistic insights and we conclude with a reflection on the main issues to be tackled in this field, which in our opinion should experience major advances in the coming years.
\end{abstract}

Departament de Quimica Física i Institut Universitari d'Electroquímica, Universitat d'Alacant, Apartat 99, E-03080, Alicante, Spain.E-mail: roberto.gomez@ua.es $\dagger$ Current address: Department of Advanced Materials for Energy, Catalonia Institute for Energy Research (IREC), Jardins de les Dones de Negre, 1, E-08930 Sant Adrià de Besòs, Barcelona, Spain.

\section{Introduction}

\section{Utilization of solar energy}

Solar light represents a clean, abundant, cheap and (virtually) endless energy source ${ }^{1,2}$ that could be used for transitioning the

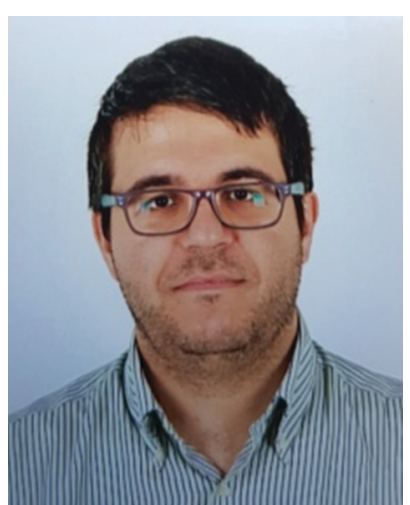

Damián Monllor-Satoca
D. Monllor-Satoca (BSc and MSc in Chemistry) received a $P h D$ in Materials Science, supervised by Prof. R. Gómez (University of Alicante, 2010). He did a Postdoctoral stay in the Pohang University of Science and Technology (POSTECH, South Korea), with Prof. W. Choi. Then, he moved back to Spain to the Catalonia Institute of Energy Research (IREC) as a Researcher with Prof. J. R. Morante, and later to the IQS-University Ramon Llull as a Lecturer. He presently works as Senior Researcher in the University of Alicante. His research interests include: (1) semiconductor photoelectrochemistry, (2) artificial photosynthesis, and (3) heterogeneous photo(electro)catalysis for environmental applications.

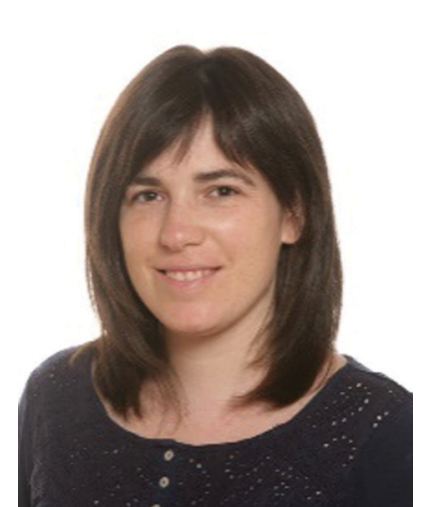

María Isabel Díez-García
M. I. Díez-García studied Chemical Engineering at the University of Alicante, where she also obtained a Master in Materials Science. In 2018, she received her $\mathrm{PhD}$ for the study of metal oxide photocathodes for solar energy conversion in the research group of "Photochemistry and Electrochemistry of Semiconductors" under the supervision of Prof. R. Gómez at the University of Alicante. She later continued for two years as a postdoctoral researcher with the same group. Most recently, she joined the Catalonia Institute for Energy Research (IREC) as a Juan de la Cierva postdoctoral fellow with Prof. J. $R$. Morante. Among her research interests are the fundamentals and applications of (photo)electrochemistry for energy conversion. 
energy needs of our society to a more environmentally friendly, less polluting and less fossil-fuel dependent economy. However, its intermittency represents a major bottleneck to its widespread use, requiring convenient materials and technologies that could efficiently absorb, use and store it. ${ }^{3,4}$ At the core of this technology is the use of semiconductor materials (photocatalysts, either as powder suspensions or thin films), characterized by an energy band gap that allows them to absorb light and generate electrons and holes that could drive charge transfer processes when interfaced with a solution with appropriate redox couples. ${ }^{3,5,6}$ Two main environmental and energy applications find their way through heterogeneous photocatalysis: ${ }^{7,8}$ (i) the degradation of noxious substances (photocatalysis), and (ii) the generation of value-added chemicals (photosynthesis). However, as Osterloh ${ }^{6}$ and Rajeshwar ${ }^{4}$ recently stated, these applications are often confused in the literature, and even the IUPAC sometimes uses misleading definitions. ${ }^{9}$

To understand their differences, we should invoke: (i) their thermodynamics, (ii) the charge transfer processes, ${ }^{10}$ and (iii) the overall energy balance. Regarding the first, a reaction energy diagram is required (Fig. 1, top); in photocatalysis, the reaction proceeds "down-hill" and the change on the reaction Gibbs energy $(\Delta G)$ is negative, thus the process is spontaneous; however, in photosynthesis the reaction proceeds "up-hill", the reaction $\Delta G$ is positive and the process non-spontaneous. ${ }^{3}$ As for the second issue, recalling the semiconductor band diagrams ${ }^{11,12}$ (Fig. 1, down), the electrons and holes are respectively transferred to electron acceptors (A) or donors (D), but their relative positions with respect to the band edges determine whether the process is photocatalytic or photosynthetic. Finally, in terms of the energy balance, ${ }^{5,6}$ the photocatalytic process uses light to accelerate the reaction rate, whereas the photosynthetic process effectively stores light energy as chemicals. In this regard, the process of artificial photosynthesis ${ }^{5}$ to store solar energy as on-demand fuels (e.g. hydrogen, methanol, etc.) is considered one of the "holy grails" in chemistry and a way to circumvent the solar intermittency.

\section{Photoelectrocatalysis}

In spite of the promising environmental applications and good prospects of heterogeneous photocatalysis, the process generally suffers from a series of demerits, including: (i) a high rate of electron-hole recombination that can be faster than their interfacial transfer rates, ${ }^{10}$ and (ii) a sluggish kinetics for the redox processes occurring on the surface of the bare photocatalyst. $^{13}$ One strategy to partially ameliorate these limitations is the application of an external potential bias with the semiconductor as a film supported on a conducting substrate (photoelectrode), thus electrochemically assisting the photocatalytic process. ${ }^{9}$ This bias is applied with respect to the counter electrode, which selectively drives one of the charge carriers out of the illuminated semiconductor film, concomitantly increasing the charge transfer rate of the carriers remaining at the surface of the semiconductor and decreasing the recombination rate. ${ }^{14}$

In comparison with conventional photocatalysis (PC), photoelectrocatalysis (PEC): (i) is performed with semiconductor films on conducting substrates, while PC can be performed with both suspensions or films on non-conducting substrates, (ii) operates under an applied external potential, while PC operates under open-circuit conditions (no external bias), (iii) anodic and cathodic processes can be individually studied or enhanced with the appropriate bias, while in PC, both processes occur simultaneously at the photocatalyst surface, (iv) the electron and hole fluxes are not the same and thus a net photocurrent is recorded, while in PC the flux of both carriers is the same, and (v) the energy efficiency is lower than in PC, as

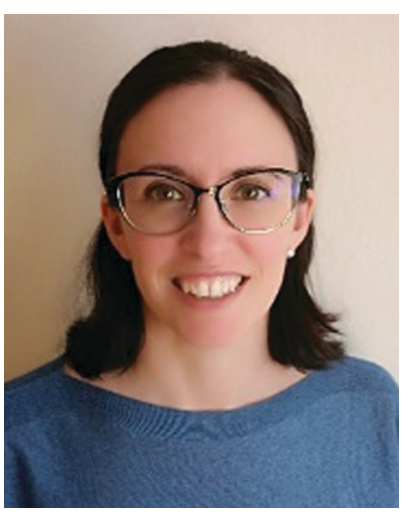

Teresa Lana-Villarreal
T. Lana-Villarreal obtained her diploma in Chemistry in 1999 from the University of Navarra (Spain). During her PhD, she mainly worked on the photooxidation mechanism of organics on $\mathrm{TiO}_{2}$ electrodes at the University of Poitiers (France) and at the University of Alicante (Spain). She made a postdoctoral stay with Prof. A. Hagfeldt at KTH (Sweden) on DSSC. Afterwards, she moved back to the University of Alicante where she joined the research group of "Photochemistry and Electrochemistry of Semiconductors" lead by Prof. R. Gómez and became associate professor in 2011. Her research activities include fundamental aspects of metal oxide semiconductors and their use in technological applications.

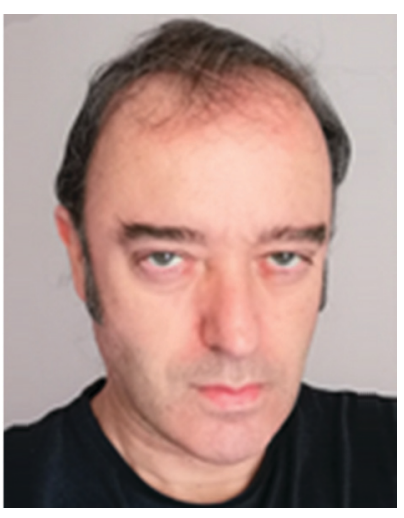

Roberto Gómez
R. Gómez obtained his PhD from the University of Alicante (Spain) in 1994 working on the Pt-group single crystal electrochemistry. After a postdoctoral stay at Purdue University (USA), he moved back to Alicante where he currently is professor of physical chemistry and leads the group of Semiconductor Photochemistry and Electrochemistry. In 1999 he was a visiting scientist at Bath University (UK) with Prof. Peter and a year later at the National

Renewable Energy Laboratory (USA) with Dr Nozik. His research interests include semiconductor electrochemistry (applied to solar fuel generation), alkaline and alkaline-earth batteries, green electrochemistry and sonochemistry. 


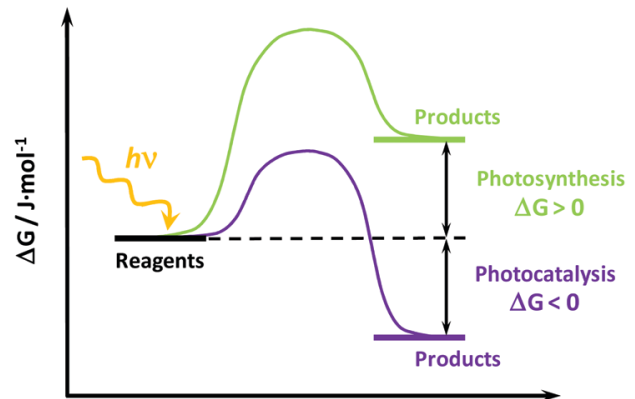

Reaction coordinate $(q)$

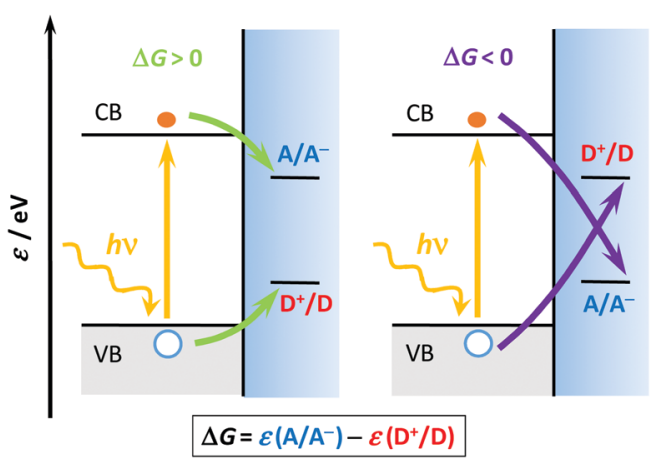

Fig. 1 Schemes for the energy vs. reaction coordinate curve (top) and the corresponding band structures (down) for two generic processes: photosynthetic (down left, green lines) and photocatalytic (down right, purple lines). Symbols: $C B$ and VB, conduction and valence bands, respectively; $A / A^{-}$, redox couple associated with the electron acceptor $(A) ; D^{+} / D$, redox couple associated with the electron donor or hole acceptor (D); $\Delta G$, Gibbs energy of reaction; $\varepsilon$, energy in the absolute scale; $h \nu$, photon energy; $q$, reaction coordinate. The equation below shows how to calculate $\Delta G$ from the energies of each redox couple. Grey and blue shaded areas represent the filled VB states and the electrolyte, respectively. The top scheme was adapted from ref. 6 , and the down scheme was adapted from ref. 11 and 12 .

the PEC bias represents an extra energy supply to drive the desired reactions.

\section{Layout of the manuscript}

In this feature article, we will revise the main contributions of the authors to the field of photoelectrocatalysis with semiconductor electrodes, in particular to produce solar fuels. We will first discuss the features of metal (hydr-)oxides as electrodes, including experimental and theoretical strategies to improve their performance. Next, we will revise the electrochemical behavior of metal oxides, both as compact or nanostructured thin films, and how electrochemical measurements can be used to elucidate their electronic structure, as well as the nature of surface and/or trap states. Then, we will review the use of binary and ternary oxides for water photooxidation (oxygen evolution reaction, OER), water photoreduction (hydrogen evolution reaction, HER) and combined in a tandem cell (full water splitting), including the use of metal hydroxides as co-catalysts; particular emphasis will be given to modeling the charge transfer processes of these systems and the mechanistic information that could be gained from them. To conclude, a reflective outlook that both accounts for the main issues that remain to be addressed and tries to shed some light on the future of this research field will also be given.

\section{The performance of metal oxides under illumination}

\section{Metal oxides and hydroxides as electrodes}

The features of the (photo)electrodes are vital to ensure that they efficiently harvest and utilize solar energy, ${ }^{15}$ while showing potential for their upscaling and implementation in practical devices. ${ }^{16}$ As such, the semiconductors to be employed must meet some stringent requirements, including: ${ }^{15,17-19}$ (i) absorb most of the useful solar spectrum, mainly composed of visible light (optical constraints); (ii) efficiently separate and transport charge carriers through the material to the appropriate interfaces (spatial charge constraints); (iii) efficiently transfer charge carriers at the semiconductor-electrolyte interface (SEI) to the appropriate redox couples (charge transfer constraints); and (iv) be inexpensive, abundant, non-toxic and durable upon many operation cycles (availability and stability constraints).

Among the most used materials in photoelectrocatalysis are metal oxides and hydroxides, ${ }^{19,20}$ both as photocatalysts and electrocatalysts (i.e. co-catalysts). ${ }^{13,21,22}$ Some of the advantages of these materials are: (i) chemical stability in different aqueous media and under illumination, (ii) appropriate position of band edges $^{15,18,19,23,24}$ for driving many redox processes, (iii) low cost, and (iv) straightforward synthesis and processing. However, they are generally limited by: ${ }^{18,19}$ (i) slow charge transfer kinetics at the surface (i.e. large overpotentials for different redox processes), (ii) relatively wide band gaps that prevent the absorption of a large portion of the solar spectrum, and (iii) large surface density of defects that portray increased charge recombination. ${ }^{20}$ Binary oxides have been by far the most studied as both photoanodes and photocathodes; ${ }^{25}$ however, ternary oxides (e.g. spinels, delafossites, perovskites and scheelites) have also emerged as promising materials due to their potentially narrow band gaps, which allow them to absorb a large portion of the visible solar spectrum. ${ }^{26}$ A detailed description of the features of different oxides and hydroxides used in (photo)electrocatalysis can be found elsewhere. ${ }^{19,20,23}$

\section{Strategies to improve the photoelectrocatalytic activity}

Prior to discussing how to improve the performance of semiconductor electrodes, we should first analyze the factors influencing their efficiency. As a reference magnitude, we shall take the potential-dependent photocurrent density $\left(j_{\mathrm{ph}}(E)\right.$, $A \mathrm{~cm}^{-2}$ ) from which the overall external or internal quantum efficiencies can be easily obtained (EQE or IQE, eqn (1) and (2) respectively):

$$
\begin{gathered}
\mathrm{EQE} \equiv \frac{j_{\mathrm{ph}}(E)}{e \Phi_{0}}=\eta_{\mathrm{abs}} \cdot \eta_{\mathrm{sep}}(E) \cdot \eta_{\mathrm{tr}}(E) \cdot \eta_{\mathrm{ct}}(E) \\
\mathrm{IQE} \equiv \frac{\mathrm{EQE}}{\eta_{\mathrm{abs}}}=\eta_{\mathrm{sep}}(E) \cdot \eta_{\mathrm{tr}}(E) \cdot \eta_{\mathrm{ct}}(E)
\end{gathered}
$$



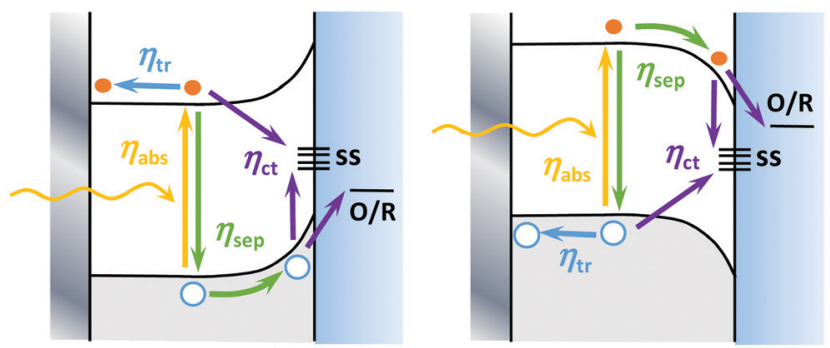

$$
\begin{gathered}
\begin{array}{c}
n \text {-type } \\
\text { (photoanode) }
\end{array} \\
\eta_{\mathrm{ats}} \\
\eta_{\mathrm{sep}}=\eta_{\mathrm{sep}}\left(k_{\mathrm{r}}, L_{\mathrm{p}}\right) \\
\eta_{\mathrm{tr}}=\eta_{\mathrm{tr}}\left(N_{\mathrm{D}}, L_{\mathrm{n}}\right) \\
\eta_{\mathrm{ct}}=\eta_{\mathrm{ct}}\left(k_{\mathrm{p}}, k_{\mathrm{r}, \mathrm{ss}}\right)
\end{gathered}
$$

(photocathode)

$$
\begin{aligned}
\eta_{\text {sep }} & =\eta_{\text {sep }}\left(k_{r}, L_{n}\right) \\
\eta_{\mathrm{tr}} & =\eta_{\mathrm{tr}}\left(N_{\mathrm{A}}, L_{\mathrm{p}}\right) \\
\eta_{\mathrm{ct}} & =\eta_{\mathrm{ct}}\left(k_{\mathrm{n}}, k_{\mathrm{r}, \mathrm{ss}}\right)
\end{aligned}
$$

Fig. 2 Scheme with the band structure and the semiconductor electrolyte interface (SEI) depicting the efficiencies $(\eta)$ for all the light-induced processes as a function of different physical magnitudes, in the case of an n-type photoanode (left) and a p-type photocathode (right). Symbols: $\eta_{\text {abs }}$ (yellow), $\eta_{\text {sep }}$ (green), $\eta_{\mathrm{tr}}$ (blue) and $\eta_{\mathrm{ct}}$ (purple) refer to the efficiencies of light absorption, charge separation, majority charge carrier transport, and minority charge carrier transfer processes, respectively; $E_{\mathrm{g}}$, band gap energy; $\alpha$, light absorption coefficient; ss, surface states; O/R, dissolved or adsorbed redox couple at the $\mathrm{SEl} ; k_{\mathrm{n}}$ and $k_{\mathrm{p}}$, minority carrier transfer rate constants for electrons and holes at the SEl, respectively; $k_{r}$ and $k_{r, s s}$ charge recombination rate constants in the bulk and at surface states, respectively; $L_{n}$ and $L_{p}$, diffusion lengths of electrons (majority carriers in photoanodes, minority carriers in photocathodes) and holes (majority carriers in photocathodes, minority carriers in photoanodes), respectively; $N_{D}$ and $N_{A}$, charge donor or acceptor densities in $\mathrm{n}$ - or $\mathrm{p}$-type semiconductors, respectively.

where $e$ is the elementary charge (C), $\Phi_{0}$ is the incident average solar photon flux $\left(\mathrm{cm}^{-2} \mathrm{~s}^{-1}\right), \eta_{\mathrm{abs}}$ is the light absorption efficiency, $\eta_{\mathrm{sep}}(E)$ is the charge separation efficiency, $\eta_{\mathrm{tr}}(E)$ is the majority charge carrier transport efficiency, and $\eta_{\mathrm{ct}}(E)$ is the minority charge carrier transfer efficiency (Fig. 2); as $\eta_{\text {abs }} \leq 1$, then EQE $\leq$ IQE. It should be noted that some authors consider $\eta_{\text {sep }}(E)$ and $\eta_{\mathrm{tr}}(E)$ conceptually assimilable, distinguishing only three efficiencies. ${ }^{27,28}$ Moreover, experimental procedures have been devised to individually determine each efficiency through the photocurrent comparison in the presence and the absence of an efficient (hole) scavenger. ${ }^{29,30}$ These overall efficiencies are also known as incident photon-to-current efficiency (IPCE for EQE) and absorbed photon-to-current efficiency (APCE for IQE), and they are used as diagnostic tools for testing the materials. ${ }^{28}$

Interestingly, it is possible to improve most of the individual efficiencies by tuning some semiconductor properties, including (Fig. 2): (i) optical properties that affect $\eta_{\mathrm{abs}}$, including the band gap width and the absorption coefficient; (ii) electronic structure, which affects $\eta_{\mathrm{sep}}(E)$ and $\eta_{\mathrm{tr}}(E)$, including the band edge positions and carrier mobility; (iii) crystallinity, which affects $\eta_{\mathrm{tr}}(E)$, including the rates of bulk recombination and diffusion-drift; and (iv) surface properties that affect $\eta_{\mathrm{ct}}(E)$, including the rates of carrier transfer and surface recombination. ${ }^{31}$ Among the experimental strategies employed to improve these efficiencies, we could briefly mention: ${ }^{32}$ (i) bulk doping to change the conductivity of the material $\left(\eta_{\mathrm{tr}}(E)\right)$ and the width of the band gap $\left(\eta_{\text {abs }}\right)$, (ii) nano- or mesostructuring to change the charge carriers' diffusion rates $\left(\eta_{\mathrm{sep}}(E)\right.$ and $\left.\eta_{\mathrm{tr}}(E)\right)$ and surface transfer rates $\left(\eta_{\mathrm{ct}}(E)\right),{ }^{23,24}$ (iii) compositing with other metals or semiconductors to generate homo- or heterojunctions to change the charge separation, transport or optical properties, (iv) surface modification $^{31}$ to change the catalytic properties $\left(\eta_{\mathrm{ct}}(E)\right)$ and light absorption, by means of: optical sensitization, passivation or protective thin layers, co-catalysts and interfacial conditioning (through the adsorption of inert ions or dipoles). ${ }^{32}$ As these strategies are beyond the scope of this article, we refer here the readers to excellent recent reviews that cover them in more depth. $^{10,13,17,27,33}$

\section{Importance of modeling}

To better guide the choice of materials and the modification strategy that could enhance the performance of the metal (hydr-)oxide (photo)electrode, it is paramount to model the (photo)electrochemical behavior of the system. ${ }^{34,35}$ This modeling can be performed at three levels: that of the materials, that of the electrode charge dynamics, and that of the full devices.

The material-level modeling is based on computational approaches to obtain the electronic structure, ${ }^{36}$ being dominant the DFT calculations. They are used in this context to identify metal and non-metal combinations (binary or multinary) yielding optimum electronic structure (band engineering) to thermodynamically and kinetically drive the desired redox processes and absorb most of the solar spectrum. It is worth noting that, together with the computational approach, a combinatorial approach can also be followed to individuate candidates for photoelectrodes, ${ }^{37}$ with a benchmarking experimental procedure that can readily map out the best elemental combination in terms of photocurrent performance.

Concerning the charge dynamics, either a microscopic or macroscopic approach has been conventionally followed. In the former case, the Marcus-Gerischer kinetic formulation ${ }^{32,38,39}$ has been mainly used, where the charge transfer rates depend on the overlapping degree between the density of states of the semiconductor and that of the redox species in the electrolyte. In the latter case, the mainstream involves solving the continuity equation for the charge carriers under appropriate boundary conditions (e.g. imposing minority charge transfer at the interface), likewise in conventional semiconductor device physics. In particular, the Gärtner formulation ${ }^{40}$ of the SEI as a Schottky junction was the founding modeling cornerstone that was later taken over with the development of nanostructured porous materials and their particular dynamics in the absence of band bending. ${ }^{41}$ In such a case, the role of surface kinetics at the SEI seems determining for the final electrode behavior. ${ }^{42,43}$ In this respect, Bisquert and coworkers ${ }^{44}$ have significantly contributed to model the performance of nanostructured electrodes in connection with impedance ${ }^{45}$ and dark voltammetric ${ }^{46}$ measurements.

As for the device level, complexity rises to a scale that requires computational fluid dynamics (CFD) and/or multiphysics simulations of all fundamental processes taking place 
simultaneously, including: ${ }^{16,47}$ light absorption, charge transport, (multiphase) mass transport, and charge migration. Regarding the charge transfer phenomena at the SEI, most studies use the Gärtner model for the photoelectrode and the Butler-Volmer equation for the electrocatalytic charge transfer (with or without co-catalysts). ${ }^{48}$ In this line, recent efforts have been devoted to modeling the performance of tandem cells where a photoanode and a photocathode are combined (see below). ${ }^{49,50}$

\section{The electrochemical response of nanostructured and compact oxides}

\section{Fundamentals}

The electrochemical behavior of a metal oxide semiconductor (SC) electrode stems from a combination of many parameters, including its chemical nature, doping degree, defects, and the electrode morphology. In this context, we can distinguish between massive compact electrodes (i.e. single crystals or compact polycrystalline electrodes) and nanocrystalline electrodes (also known as mesoporous, mesoscopic, nanoporous or nanostructured electrodes), in which the electrolyte can penetrate/permeate the material. They consist of a porous film of nanocrystals deposited on a conducting substrate, where the nanoparticles are electrically interconnected between them and with the conducting substrate. As the reactions of interest occur at the illuminated SEI, these electrodes can be advantageous under certain circumstances.

Due to the small nanoparticle size, low doping degree and the percolation of the electrolyte throughout the porous thin film, such electrodes are not able to sustain a significant electric field or band bending. ${ }^{51,52}$ This clearly contrasts with the situation of single crystals or bulk polycrystalline electrodes in which charge separation relies on a built-in electric field. ${ }^{53}$ In the case of nanoporous electrodes, one of the photogenerated charge carriers is preferentially consumed at the SEI, inhibiting their recombination; thus, the charge-separation occurs mainly due to the different kinetics of the processes involving the consumption of photogenerated electrons and/or holes, defining its n-type (photoanode) or p-type (photocathode) character. On the other hand, the charge carrier concentration gradient promotes their transport by diffusion; ${ }^{51,52}$ the charge carrier displacement through the electrode induces the concomitant displacement of ions from the electrolyte to compensate the charge and to keep the electroneutrality of the system. Some authors refer to this transport mechanism as ambipolar diffusion. ${ }^{54}$

Titanium dioxide is the prototypical n-type SC reference material. In this case, photogenerated holes usually are easily consumed or trapped at the surface, while electrons can diffuse though the electrode to the back-contact, provided the substrate Fermi level (potential) is sufficiently low (high). ${ }^{55}$ Although this general qualitative behavior is conventionally considered in the scientific literature, there are some controversial issues that limit reliable predictions of the behavior of new synthetic materials or the interpretation of some (photo)electrochemical results. This paucity of information encouraged us some years ago to study and to model the (photo)electrochemical behavior of nanoporous $\mathrm{TiO}_{2}$ electrodes. In this regard, we believe that the electrochemical measurements in the dark in the absence of electroactive species can be cautionary used as the fingerprint of the material's density of states (DOS), while the (photo)electrochemical measurements in the presence of electroactive species can be used to obtain further information on its photo(electro)catalytic activity. ${ }^{32,56}$

In particular, the cyclic voltammetry of metal oxide electrodes in the absence of faradaic processes shows capacitive currents. Such capacitive currents arise by a series connection of two capacitances $\left(\mathrm{F} \mathrm{cm}^{-2}\right)$, one associated with the solid ( $\left.C_{\text {solid }}\right)$ and the other with the compact double layer at the SEI, i.e. the Helmholtz layer $\left(C_{\mathrm{H}}\right)$ (eqn (3)):

$$
\frac{1}{C}=\frac{1}{C_{\text {solid }}}+\frac{1}{C_{\mathrm{H}}}
$$

In the case of low-doped bulk SCs in contact with an electrolyte, the Fermi level equilibration of the material and that of the electrolyte induces the band bending phenomenon and the concurrent formation of a space charge region (SCR). If $C_{\text {solid }} \ll C_{\mathrm{H}}$, a change of the electrode potential has the effect of shifting the Fermi level in the SCR with respect to the energy levels in the solid, whereas the energy levels of the solid are pinned at the surface (band edge pinning). In this case, $C_{\text {solid }}$ can be directly associated with the capacitance per electrode unit area of the SCR, $\left(C_{\mathrm{SC}}\right)$. This capacitance can be obtained by solving the Poisson-Boltzmann equation; for an n-type SC under depletion conditions, the potential dependence of $C_{\mathrm{SC}}$ is given by the Mott-Schottky equation, which is usually employed to determine the flat band potential $\left(E_{\mathrm{FB}}, \mathrm{V}\right)$ and the bulk donor density $\left(N_{\mathrm{D}}, \mathrm{cm}^{-3}\right)$ of the material (eqn (4)):

$$
\frac{1}{C_{\mathrm{SC}}^{2}}=\frac{2}{e \varepsilon \varepsilon_{0} N_{\mathrm{D}}}\left(E-E_{\mathrm{FB}}-\frac{k_{\mathrm{B}} T}{e}\right)
$$

where $\varepsilon$ is the SC relative dielectric constant, $\varepsilon_{0}$ is the vacuum permittivity $\left(8.85 \times 10^{-14} \mathrm{~F} \mathrm{~cm}^{-1}\right), k_{\mathrm{B}}$ is the Boltzmann constant $\left(1.38 \times 10^{-23} \mathrm{~J} \mathrm{~K}^{-1}\right), T$ is the absolute temperature $(\mathrm{K}), e$ is the elementary charge $\left(1.602 \times 10^{-19} \mathrm{C}\right)$, and $E$ is the applied potential $(\mathrm{V})$.

In this context, to apply eqn (4), the electrode should sustain a built-in potential, which requires the building blocks constituting the electrodes to be large enough. Porosity can be corrected by considering the real surface area. ${ }^{57}$ However, in the literature we can find examples were electrochemical impedance spectroscopic (EIS) measurements have been employed to experimentally obtain the capacitance of nanoporous electrodes and determine $N_{\mathrm{D}}$, without considering that the real surface area might be orders of magnitude larger than the geometric one. ${ }^{58}$ 
Electronic structure of nanoporous electrodes: surface states and trap states

The behavior of nanoporous electrodes cannot be rationalized on the basis of the existence of a SCR. In this case, when the Fermi level (potential) is far below (above) the conduction band edge (CB), they are insulating; as it approaches the CB edge upon negative polarization, within a potential range the electrode exhibits a certain degree of conductivity, which allows for a homogeneous charging of the electrode and thus for a homogeneous shift of the Fermi level with respect to the $\mathrm{CB}$, provided the band edges are pinned. The conductivity arises from the thermal excitation of electrons to the $\mathrm{CB}$ or other states near the $\mathrm{CB}$, or even by hopping between different electronic states. This causes a homogeneous electron occupancy of band-gap states, levelling with the substrate potential. The $C_{\text {solid }}$ can be related in this case with the intrinsic film capacitance or chemical capacitance $\left(C_{\mu}\right)$, which usually is much smaller than $C_{\mathrm{H}}$ and dominates the final electrochemical behavior. Neglecting the contribution coming from the SEI, and assuming an ideally polarizable electrode in quasi-equilibrium with band edge pinning conditions, $C_{\mu}$ (per geometric unit area) can be defined as (eqn (5)): ${ }^{59}$

$$
C_{\mu}=p e^{2} L \frac{\mathrm{d} n}{\mathrm{~d} \varepsilon_{\mathrm{F}}}=p e^{2} L D\left(\varepsilon_{\mathrm{F}}\right)
$$

where $p$ is the porosity of the film, $L$ is the film thickness $(\mathrm{cm})$, $n$ is the electron density $\left(\mathrm{cm}^{-3}\right), \varepsilon_{\mathrm{F}}$ is the Fermi level $(\mathrm{eV})$, and $D\left(\varepsilon_{\mathrm{F}}\right)$ is the density of states $\left(\mathrm{cm}^{-3} \mathrm{eV}^{-1}\right)$. As the Fermi level can be modified by changing the applied potential with respect to a reference electrode $\left(\mathrm{d} \varepsilon_{\mathrm{F}}=-e \cdot \mathrm{d} E\right)$, this opens up the possibility of

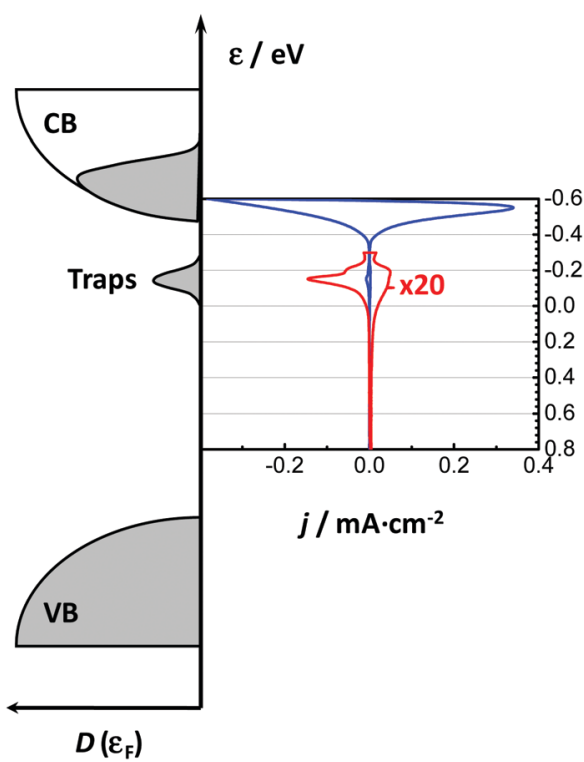

Fig. 3 Scheme of the correlation between the cyclic voltammogram obtained in the dark in the absence of faradaic currents and the electronic density of states (DOS) for a nanoporous rutile electrode. VB and CB represent the valence and conduction band, respectively. The blue line corresponds to the filling/emptying of $\mathrm{CB}$ while the red line (intentionally magnified) refers to the filling/emptying of monoenergetic trap states at the grain boundaries. extracting information on the density of electrochemically accessible electronic states in a SC electrode, by measuring the capacitance as a function of potential.

Bisquert and co-workers were the first to show the potentialities of cyclic voltammetry for this purpose. ${ }^{46,59-62}$ The capacitive currents measured can be ascribed as the filling/emptying of electronic states. The capacitive current density $\left(j, \mathrm{~A} \mathrm{~cm}^{-2}\right)$ relates the change of electron density with the change of electrode potential according to (eqn (6)):

$$
j=C v=e L v \frac{\mathrm{d} n}{\mathrm{~d} E}
$$

where $v$ is the potential scan rate $\left(\mathrm{V} \mathrm{s}^{-1}\right)$. Accordingly, the current measured at a certain potential directly gives us the variation of the number of states with the potential (Fig. 3). The remaining question is the physical location of these electronic states; according to Bisquert and co-workers, ${ }^{46,59-62}$ for $\mathrm{TiO}_{2}$ these states correspond to $\mathrm{CB}$ and to an exponential distribution of surface states (SS) below the CB.

In line with the above ideas, we reviewed the variation of the capacitance with the potential for different locations of the electronic states. ${ }^{32,56}$ Considering eqn (5) and (6), and the theoretical DOS in the CB for an n-type SC, two limiting cases for the capacitance due to $\mathrm{CB}$ states can be obtained: (i) for a non-degenerated SC (eqn (7)):

$$
C_{\mu}^{\mathrm{CB}}=p \frac{e^{2} N_{\mathrm{c}} L}{k_{\mathrm{B}} T} \exp \left(\frac{e\left(E_{\mathrm{c}}-E\right)}{k_{\mathrm{B}} T}\right)
$$

and (ii) for a degenerated SC, under strong accumulation conditions (eqn (8)):

$$
C_{\mu}^{\mathrm{CB}}=\frac{2 p}{\sqrt{\pi}} \frac{e^{2} N_{\mathrm{c}} L}{k_{\mathrm{B}} T}\left(\frac{e\left(E_{\mathrm{c}}-E\right)}{k_{\mathrm{B}} T}\right)^{1 / 2}
$$

where $N_{\mathrm{c}}$ represents the CB states per unit volume $\left(\mathrm{cm}^{-3}\right)$ and $E_{\mathrm{c}}$ is the CB edge potential $(\mathrm{V})$. In the case of a non-degenerated $\mathrm{SC}$, by representing $\ln C_{\mu}^{\mathrm{CB}}$ versus $E$, a straight line with a slope of $\left(-e / k_{\mathrm{B}} T\right)$ is obtained, where the location of the $\mathrm{CB}$ can be estimated from the $y$-axis intercept. ${ }^{56}$

Considering now the intra-band gap states below the CB (i.e. surface states), at the zero-limit temperature, the corresponding capacitance can be estimated as (eqn (9)): ${ }^{46}$

$$
C_{\mu}^{\mathrm{SS}}=\frac{e^{2} \Theta_{\mathrm{t}} L}{k_{\mathrm{B}} T_{\mathrm{C}}} \exp \left(\frac{e\left(E_{\mathrm{c}}-E\right)}{k_{\mathrm{B}} T_{\mathrm{C}}}\right)
$$

where $\Theta_{\mathrm{t}}$ is the electrode total volume density of traps $\left(\mathrm{cm}^{-3}\right)$, and $T_{\mathrm{C}}$ a characteristic temperature that defines the broadening of the exponential trap distribution. In addition to a surface-state distribution in the band gap, some authors ${ }^{63-66}$ have proposed the existence of monoenergetic states or traps in the band gap. For these monoenergetic states the associated chemical capacitance can be described by (eqn (10)):

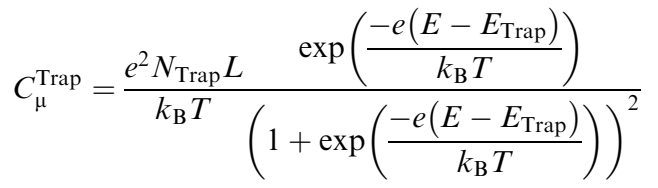


where $N_{\text {Trap }}$ is the density of defined monoenergetic states $\left(\mathrm{cm}^{-3}\right)$, and $E_{\text {Trap }}$ their location in the potential scale (V). Considering all the contributions from CB electrons, surface-state electrons and electrons trapped at monoenergetic states, the total chemical capacitance for a nanoporous electrode is given by (eqn (11)):

$$
C_{\mu}=C_{\mu}^{\mathrm{CB}}+C_{\mu}^{\mathrm{SS}}+C_{\mu}^{\mathrm{Trap}}
$$

Once the theoretical ground was set, we carefully analysed the cyclic voltammograms for a series of well-defined nanoporous $\mathrm{TiO}_{2}$ electrodes. Our measurements corroborate the presence of an exponential state distribution below the $\mathrm{CB}$ for anatase, which seems to be absent in the case of rutile. ${ }^{56}$ Regarding the monoenergetic electronic states or traps, we have physically identified them as particle-particle interfaces, (i.e. grain boundaries) acting as electron traps. ${ }^{67-70}$ These states seem to play a key role as recombination centres and are highly dependent on the crystal structure and self-assembly (i.e. morphology) of the electrode, as well as on the electrolyte composition. ${ }^{15,16}$ Moreover, using different types of samples we unequivocally showed that the capacitance (i.e. the area of the cyclic voltammogram) is proportional to the real electroactive area and not to the electrode volume. ${ }^{32,69,71,72}$

It is important to mention that to counterbalance the accumulated charge and preserve the electroneutrality of the material, ions are required. For example, when electrons are accumulated in $\mathrm{TiO}_{2}$, cations get adsorbed or inserted into the crystal structure, probably limited to the external layer exposed to the electrolyte. This fact can affect the direct quantitative interpretation of the electrochemical capacitance as a direct measurement of the DOS. Only in the case of electrodes with extremely small nanoparticles in which the electrolyte can easily permeate, the whole volume may accumulate charge. ${ }^{56}$ Such ion insertion has been evinced for different metal oxide SCs and it has been shown that it could be faster than the extraction one. Therefore, it is possible to take advantage of this process to promote their photocatalytic activity, as it causes a non-permanent electrochemical doping of the material. ${ }^{67,73,74}$

Furthermore, in the case of nanoporous electrodes, the voltammetric measurements in the dark can also be combined with open circuit photopotential decay measurements. ${ }^{75}$ Such a combination allows to evaluate the recombination and transfer (to solution) rate constants of charge carriers accumulated upon illumination at the metal oxide SC electrode. This evaluation is based on the fact that the integration of the voltammetric profile provides the actual charge carrier concentration in the nanostructured film as a function of the applied potential. As the photopotential decay curve displays the potential as a function of time, by a proper combination of both measurements the time evolution of the electron concentration is obtained, and hence the corresponding kinetic rate constants. $^{75,76}$

Apart from the potential decay measurements, the currents under illumination (i.e. photocurrents) in the presence of electroactive species can also be employed to obtain further information about the energy location of the conduction band, ${ }^{56}$ the photocatalytic activity, ${ }^{69}$ the charge transfer rate and even, the reaction mechanism. ${ }^{39,77}$ In the case of $\mathrm{TiO}_{2}$, the electron donor species present in the electrolyte can be oxidized (i) indirectly, where the oxidation process occurs via surface trapped holes, also called hydroxyl radicals (by an isoenergetic interfacial hole transfer according to the Marcus-Gerischer energy model), or (ii) directly through its reaction with photogenerated VB free holes (by an inelastic interfacial hole transfer). By measuring the photocurrent as a function of the electron donor species concentration present in the electrolyte, it is possible to distinguish between both mechanisms. ${ }^{39,77}$ It is important to bear in mind that the electrochemical measurements suffer from the lack of specificity that can be surmounted by coupling them with spectroscopic measurements such as Raman or IR spectroscopy. ${ }^{78-83}$

Finally, it is worth mentioning that there exists a direct relationship between the appearance of capacitive currents in nanostructured $\mathrm{TiO}_{2}$ and $\mathrm{WO}_{3}$ electrodes and their electrocatalytic activity for cathodic processes in the dark. Our recent studies on $\mathrm{CO}_{2}$ reduction on these materials in acetonitrile solutions constitute clear examples of this behavior. ${ }^{84,85}$

\section{Water photosplitting with metal oxides}

\section{Water photooxidation}

The seminal work of Fujishima and Honda ${ }^{86}$ set the ground for using semiconductor metal oxides (i.e. $\mathrm{TiO}_{2}$ ) as photoelectrodes to sustain water oxidation in photoelectrocatalytic devices. Likewise, the works of Grätzel et al. ${ }^{23,87}$ with visible-sensitized mesoporous $\mathrm{TiO}_{2}$ as solar cells prompted the research on nanostructured materials. These breakthrough contributions inspired the experimental work on artificial photosynthesis that has continued to date. In this regard, the recent work on water photooxidation from the authors deals with the common topics in the field, including: materials, covering binary oxides (e.g. $\mathrm{TiO}_{2}{ }^{56,70,88-91} \mathrm{WO}_{3}{ }^{92-94}$ and $\mathrm{Fe}_{2} \mathrm{O}_{3}{ }^{57,91,96-98}$ ), hydroxides $\left(\mathrm{Ni}(\mathrm{OH})_{2}\right)^{99-101}$ and ternary oxides $\left(\right.$ e.g. $\mathrm{BiVO}_{4},{ }^{100-102} \mathrm{FeVO}_{4},{ }^{101}$ and $\mathrm{TaON}^{90}$ ); and modification strategies, such as doping, ${ }^{57,88,89,97,98}$ passivation, ${ }^{90,93,94}$ heterojunctions, ${ }^{91}$ compositing, ${ }^{89}$ surface modification, ${ }^{88,90,96}$ and the use of co-catalysts. ${ }^{95,99-101,103}$ In the following, we shall revise some of these works in terms of the modification strategy employed to improve the water photooxidation process (i.e. bulk modification and heterojunctions, surface modification, and the use of co-catalysts).

Bulk modification and heterojunctions. Herein, we highlight recent works that mainly change any intrinsic property of the material, such as the density of majority charge carriers (by doping) or their transport properties (through heterojunctions or compositing with other materials). Regarding the latter case, Monllor-Satoca et al. ${ }^{91}$ studied with voltammetric and impedance measurements the effect of titanium doping on the photoactivity improvement of hematite photoanodes (Fig. 4), by means of compositing hematite (host) with titania (guest) at different loading levels up to the solid state solubility limit of both components (0-20 mol\%) and with a $10-15 \%$ optimum doping range; this approach was unprecedented ${ }^{104}$ and allowed 
a.

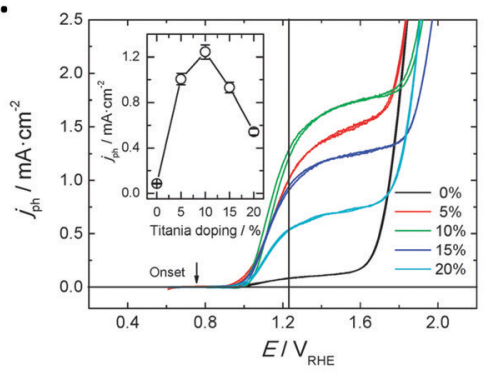

b.

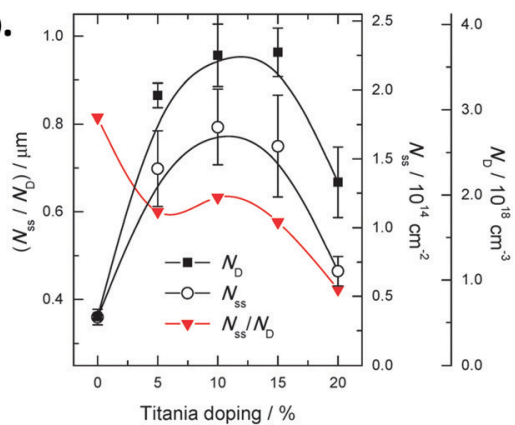

C.

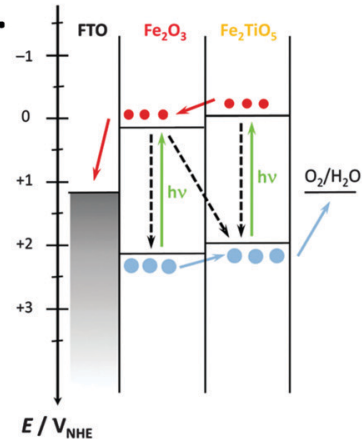

d.

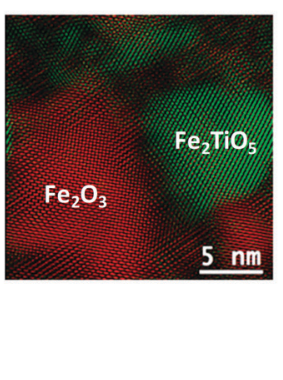

e.

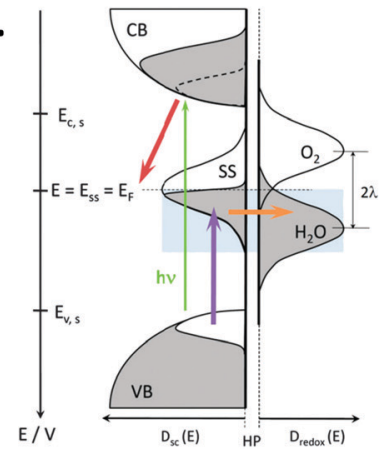

Fig. 4 (a) Voltammetric measurements for hematite/titania electrodes under illumination with different titania doping; the vertical line depicts the thermodynamic potential value for the oxygen evolution reaction $\left(1.23 \mathrm{~V}_{\mathrm{RHE}}\right)$; the inset shows the photocurrent density values at $1.23 \mathrm{~V}_{\mathrm{RHE}}$ as a function of titania doping. (b) Donor density $\left(N_{\mathrm{D}}\right)$, total surface state density $\left(N_{\mathrm{ss}}\right)$ and their ratio $\left(N_{\mathrm{ss}} / N_{\mathrm{D}}\right)$ as a function of titania doping; $N_{\mathrm{D}}$ was estimated from the slopes of the respective Mott-Schottky plots in the dark; $N_{\mathrm{ss}}$ was obtained from integration of the density of surface states profiles, estimated from impedance measurements under illumination. (c) Thermodynamic scheme of the charge generation, transfer and recombination processes at the biased $\left(1.23 \mathrm{~V}_{\mathrm{RHE}}\right)$ hematite/titania electrode under illumination, for intermediate and optimum titania doping values (5-15\%). (d) HR-TEM micrograph of the hematite/titania $20 \%$ sample, shown as a coloured inverse FFT image highlighting the hematite $\left(\mathrm{Fe}_{2} \mathrm{O}_{3}\right.$, red) and pseudo-brookite $\left(\mathrm{Fe}_{2} \mathrm{TiO} \mathrm{O}_{5}\right.$, green) crystallographic phases. (e) Kinetic scheme of the charge generation and transfer processes at the biased (1.23 $\left.\vee_{\mathrm{RHE}}\right)$ hematite/titania-electrolyte interface under illumination, for the optimum titania doping values (10-15\%). Reprinted with permission from ref. 91 . Copyright 2015 The Royal Society of Chemistry.

to gain a wider mechanistic vision of all the factors that have a role in the photoactivity improvement (i.e. 15-fold increase in photocurrent - Fig. 4a - and a 100-fold decrease in charge transfer resistance, both at $1.23 \mathrm{~V}_{\mathrm{RHE}}$ - thermodynamic potential for the oxygen/water redox couple). In particular: (i) surface and charge donor density of states (Fig. 4b), where its optimal ratio allowed both a good material conductivity and a better hole trapping-transfer kinetics at the SEI (Fig. 4e), and (ii) hematite-pseudobrookite (Fig. 4c and d) and titania-pseudobrookite heterojunctions, where the former portrays a beneficial cascade charge transfer effect, while the latter has a deleterious "hole mirror" effect. ${ }^{105}$

As for doping, recent work has been directed to the improvement of both binary and ternary oxides. In this way, Cots et al. have studied the doping of hematite with Mo, ${ }^{57,97}$ which was shown to be compatible with modification (surface passivation) with Yb. ${ }^{98}$ Quiñonero et al. ${ }^{100}$ attempted the improvement of bismuth vanadate photoanodes by means of La- or Ce-doping, and its subsequent surface modification with Au nanoparticles, as the bare material is recognized to suffer from slow charge transport and transfer rates at the SEI. ${ }^{106}$ With a 1 at $\%$ and 2 at\% of $\mathrm{La}$ and $\mathrm{Ce}$, water photooxidation was 2.3- and 4-times increased, respectively; this enhancement was probably due to the passivation of surface states, and the likeliness of lanthanum and cerium oxygenated species present at the surface that could yield a more negatively charged surface, as manifested by a negative shift on the flat band potential in both cases. Further modification with $\mathrm{Au}$ nanoparticles led to an enhanced photocurrent as they act as co-catalysts with no surface plasmon resonance effect, as shown from a direct comparison between the absorption spectra and the IPCE curves of the modified electrodes, in which no photocurrent arises in the plasmonic band region (ca. $580 \mathrm{~nm}$ ). Interestingly, this double bulk-surface modification yields photoanodes that are potentially viable in bias-free tandem devices.

Another interesting dual bulk-surface modification example is that from Kim et al. ${ }^{90}$ where titania nanotube electrodes are both N-doped and surface modified with a TaON shell, which synergistically improves the photocurrent through an enhanced visible activity and a passivation of deleterious surface states induced by $\mathrm{N}$-doping.

Surface modification. Now we shall present some works related with changes at the SEI (i.e. interface conditioning) that affect the charge transfer properties of the material, for instance through (ionic or dipole) adsorption or deposition of conformal (passivating) overlayers. Among the adsorption-based methods, one of the most studied has been the fluorination of titanium dioxide in photo(electro)catalysis; ${ }^{107,108}$ in this regard, 
we extensively studied the photoelectrochemical behavior of surface fluorinated nanoporous $\mathrm{TiO}_{2}$ electrodes. ${ }^{75,88}$ In particular, we demonstrated the effect of fluoride adsorption on the voltammetric behavior of titania electrodes both in the dark and under illumination (Fig. 5). ${ }^{88}$

In the dark, fluorination induces an increased capacitance in the accumulation potential region (Fig. 5a) for all $\mathrm{TiO}_{2}$ electrodes tested, due to the band edge level pinning phenomenon that favors proton insertion in the $\mathrm{TiO}_{2}$ particles without a significant change in the Helmholtz layer potential drop (Fig. 5e). On the contrary, the behavior under illumination is dependent on the $\mathrm{TiO}_{2}$ sample used and the substrate degraded. Fluorination increased the photocurrent for water (Fig. 5b and c) as well as for the oxidation of methanol, phenol and catechol (Fig. 5d), but decreased it for the oxidation of formic acid. The observed behavior could be rationalized in terms of the Direct-Indirect mechanism mentioned above. ${ }^{39,109}$ Substrates that do not strongly adsorb on titanium dioxide show a reduced recombination rate and an enhanced indirect oxidation rate by surface trapped holes (i.e. methanol, water). In contrast, those strongly chemisorbed show a diminished direct oxidation rate by valence band holes (i.e. formic acid), as fluorination displaces the substrates from the surface. In the case of phenol and catechol (Fig. 5d), fluoride prevents surface poisoning by oxidation intermediates, thus yielding enhanced photocurrents.
Another surface modifier that has been proven effective is trimethyl aluminum. In fact, hematite electrodes were shown to display an enhancement of the photoelectrochemical properties toward water oxidation upon modification. ${ }^{96}$ The effect was attributed to an improvement in charge carrier transport based on synchrotron radiation photoemission and X-ray absorption spectroscopy results.

Other examples of surface modification show an increased photoactivity, either through core-shell systems ${ }^{89}$ or conformal overlayers. $^{93}$ In the former case, Kim et al. ${ }^{89}$ showed that compositing titania with graphene was beneficial for the degradation of pollutants and water oxidation, as graphene enhanced charge separation. In the latter case, Kim et al. ${ }^{93}$ uncovered, through voltammetric and transient spectroscopic measurements, the role of alumina overlayers on tungsten oxide as passivation layers for water photooxidation.

Co-catalysts. Finally, we will review a particular case of surface modification in which a new catalyst is incorporated on the surface of the photoanode to promote processes that portray sluggish kinetics with the bare photoactive material, such as the OER. In particular, we will cover the use of nickel hydroxide (both as a standalone electrocatalyst ${ }^{99}$ and a co-catalyst with vanadate photoanodes ${ }^{101}$ ) and molybdenum sulfide with $\mathrm{TiO}_{2}{ }^{103}$ Cibrev et al. ${ }^{99}$ prepared and studied the electrochemical performance of ultrathin and transparent $\mathrm{Ni}(\mathrm{OH})_{2}$ layers
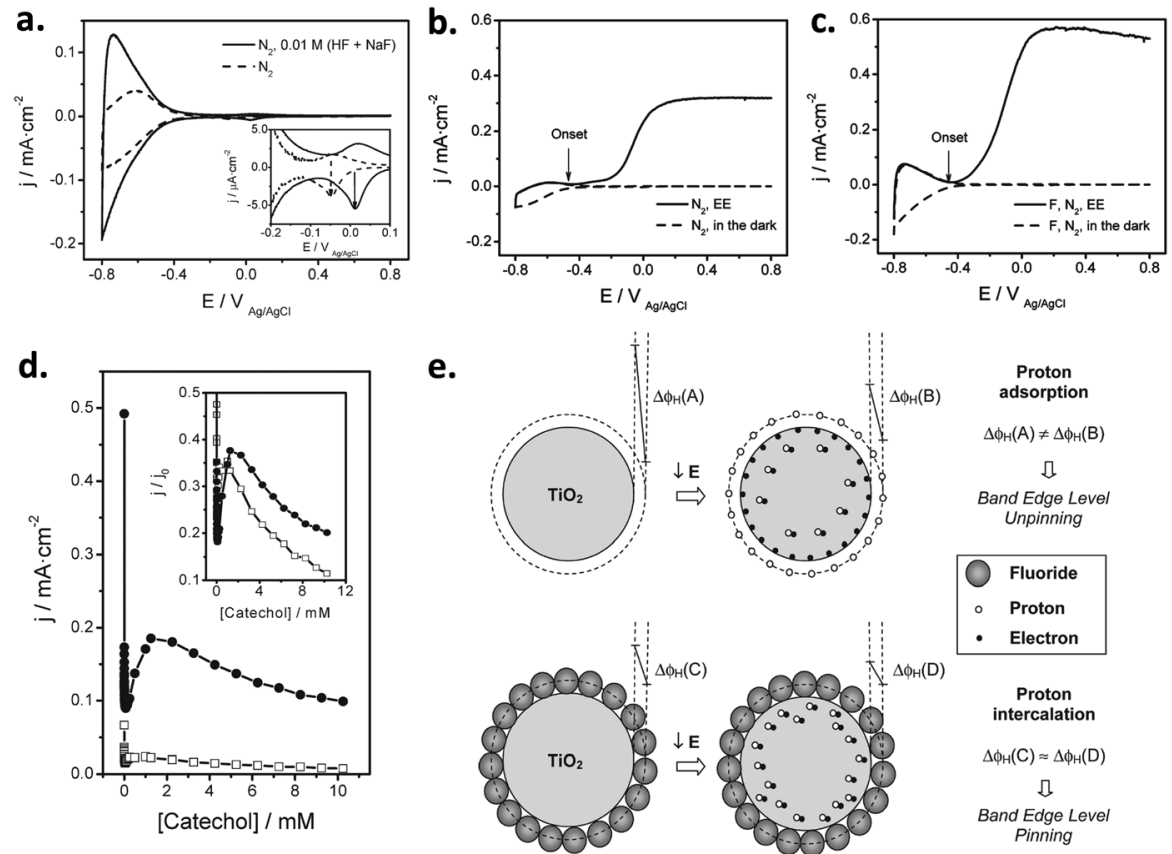

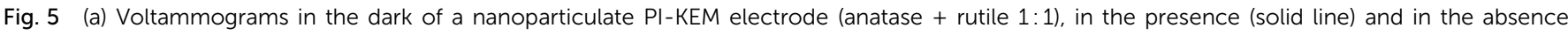

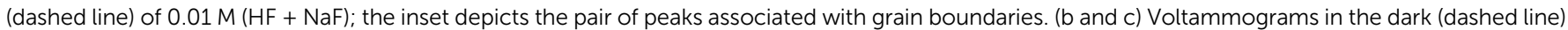

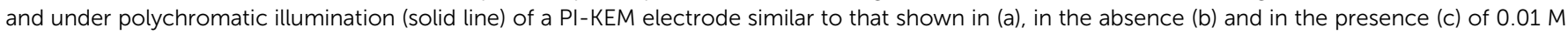

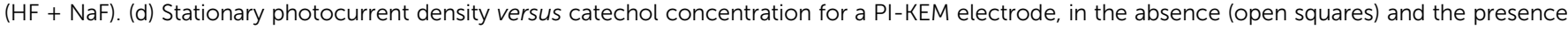

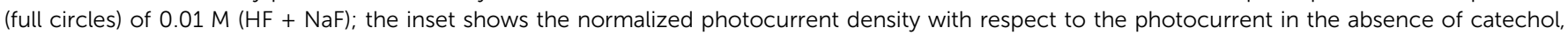

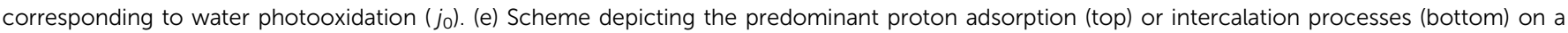

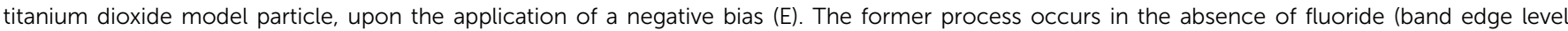

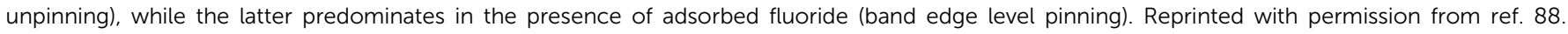
Copyright 2011 American Chemical Society. 
deposited on conducting glass (i.e. FTO, Fig. 6a-c). We observed that the catalytic activity (i.e. turnover number) for the OER could be varied at will and in a wide range of values (over 7 orders of magnitude), showing an almost inverse dependence with the amount of catalyst deposited (Fig. 6d). Interestingly, we deduced that the optimum activity occurred for $1 \mathrm{~nm}$-thick layer of $\mathrm{Ni}(\mathrm{OH})_{2}$ and thicker films did not show an improved electrocatalytic activity. These results open up the preparation of efficient transparent films of co-catalysts over photoanodes, which could be interesting in practical photoelectrochemical devices. Similar results have been obtained for $\mathrm{Fe}(\mathrm{OH})_{2}$ and $\mathrm{Co}(\mathrm{OH})_{2}$ ultrathin layers deposited on conducting glass. ${ }^{110}$

Subsequently, Quiñonero et al. ${ }^{101}$ optimized the amount of $\mathrm{Ni}(\mathrm{OH})_{2}$ to be deposited as OER co-catalyst on $\mathrm{BiVO}_{4}$ and $\mathrm{FeVO}_{4}$ photoanodes (Fig. 7). Using dark voltammetry, we were able to estimate the amount of deposited co-catalyst, showing that the photoactivity of the vanadate-based photoanodes reached an optimum with only 0.46 and 1.68 monolayers of $\mathrm{Ni}(\mathrm{OH})_{2}$ on $\mathrm{BiVO}_{4}$ and $\mathrm{FeVO}_{4}$, respectively (Fig. 7a and b); similar optimum monolayer amounts were required for $\mathrm{La}-$ and Ce-doped $\mathrm{BiVO}_{4}$ photoanodes. In all cases, the $\mathrm{Ni}(\mathrm{Iv})$ species were the actual electrocatalytic centers, generated upon a successive 2 hole capture by $\mathrm{Ni}(\mathrm{II})$; then, $\mathrm{Ni}(\mathrm{IV})$ species transfer the holes to oxidize water, catalytically re-generating $\mathrm{Ni}(\mathrm{II})$ species on the surface (Fig. 7c). In this line, Jeon et al. ${ }^{95}$ have very recently published an example of a catalytic cycle mediated by $\mathrm{Ag}(\mathrm{I})$ species adsorbed on a $\mathrm{WO}_{3}$ photoanode, acting as a hole scavenger species that upon illumination reversibly transferred the photogenerated hole to water, re-generating $\operatorname{Ag}(\mathrm{I})$ in a
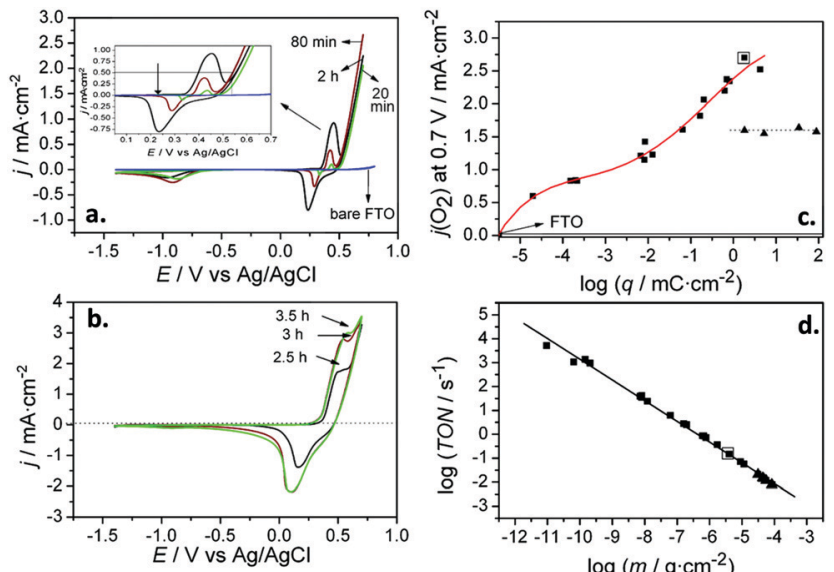

Fig. 6 ( $a$ and b) Cyclic voltammograms for bare FTO (blue line) and FTO/ $\mathrm{Ni}(\mathrm{OH})_{2}$ electrodes, corresponding to deposition times of (a) $20 \mathrm{~min}$, $80 \mathrm{~min}$ and $2 \mathrm{~h}$ (green, red, and black lines, respectively) and (b) $2.5 \mathrm{~h}$, $3 \mathrm{~h}$ and $3.5 \mathrm{~h}$ (black, red, and green lines, respectively). Inset in (a): Detail of the voltammetric curves in the high potential region. (c) Current density for the oxygen evolution reaction (OER) at $0.7 \mathrm{~V}$ for $\mathrm{FTO} / \mathrm{Ni}(\mathrm{OH})_{2}$ electrodes versus the charge density for the reduction of $\mathrm{NiOOH}$. (d) Turnover number (TON) for the OER at $0.7 \vee$ for $\mathrm{FTO} / \mathrm{Ni}(\mathrm{OH})_{2}$ electrodes as a function of the $\mathrm{Ni}(\mathrm{OH})_{2}$ deposited mass per unit geometric surface area. Reprinted with permission from ref. 99. Copyright 2012 Hydrogen Energy Publications, LLC. Published by Elsevier Ltd.
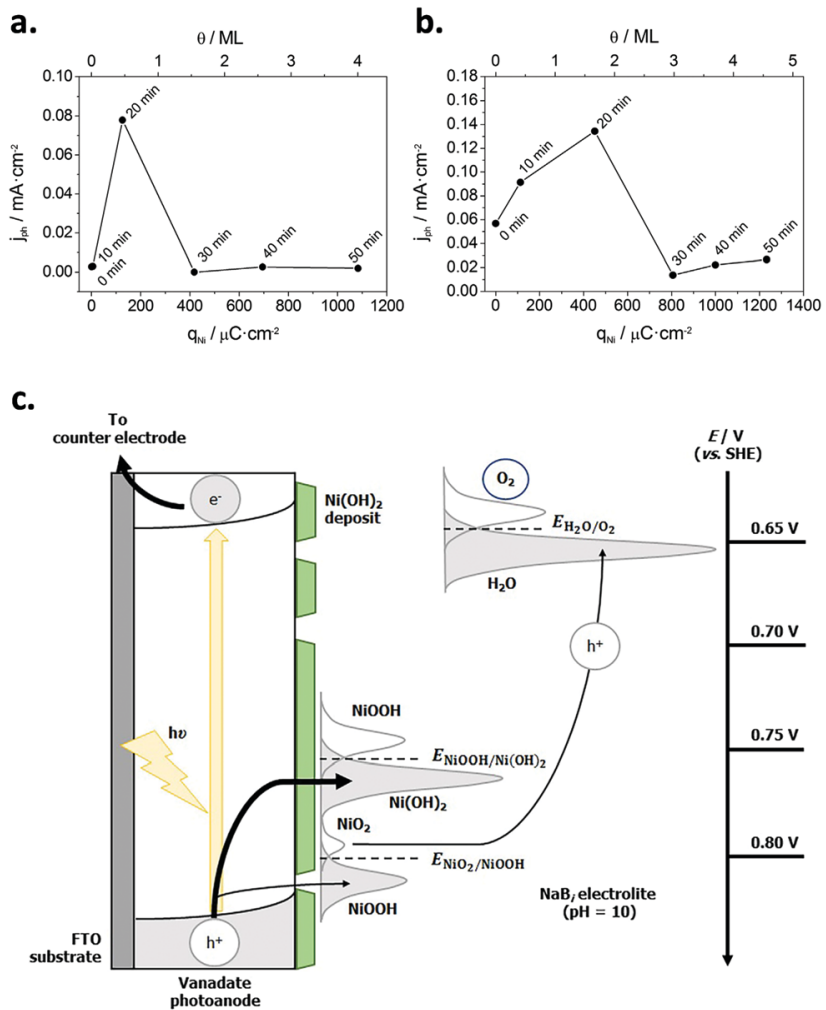

Fig. 7 (a) Photocurrent density for the oxygen evolution reaction (OER) at $-0.5 \mathrm{~V}$ for $\mathrm{BiVO}_{4} / \mathrm{Ni}(\mathrm{OH})_{2}$ photoanodes, prepared with different $\mathrm{Ni}(\mathrm{OH})_{2}$ deposition times $(0-50 \mathrm{~min}$ ) versus the charge density corresponding to the reduction of $\mathrm{NiOOH}\left(q_{\mathrm{Ni}}\right)$ and the $\mathrm{Ni}(\mathrm{OH})_{2}$ coverage $(\theta)$ on the electrode surface. (b) Photocurrent density for the OER at $0.1 \mathrm{~V}$ for $\mathrm{FeVO}_{4} / \mathrm{Ni}(\mathrm{OH})_{2}$ photoanodes, prepared with different $\mathrm{Ni}(\mathrm{OH})_{2}$ deposition times $(0-50 \mathrm{~min}) v \mathrm{~s}$. $q_{\mathrm{Ni}}$ and $\theta$. (c) Diagram for the mechanism through which the $\mathrm{Ni}(\mathrm{OH})_{2}$ deposit acts as a co-catalyst for the OER. Reprinted with permission from ref. 101. Copyright 2017 Elsevier B. V.

continuous redox cycle, only limited by the photoreduction of $\mathrm{Ag}(\mathrm{I})$ to $\mathrm{Ag}(0)$.

To conclude, we will discuss the work of Recatala et al. ${ }^{103}$ who prepared molybdenum sulfide clusters $\left(\mathrm{Mo}_{3} \mathrm{~S}_{7}\right)$ from the respective Mo complexes adsorbed on titanium dioxide; upon illumination, this hybrid system was reduced to generate $\mathrm{Mo}_{3} \mathrm{~S}_{7}$, which subsequently acted as HER co-catalyst. This system showed a reduced overpotential for hydrogen evolution (up to $300 \mathrm{mV}$ for $1 \mathrm{~mA} \mathrm{~cm}^{-2}$ ), and a significant increase of the hydrogen photogenerated in the presence of sacrificial hole scavengers (i.e. sulfide and sulfite). From a practical perspective, this system represents a simple and controlled modification method that could be used with other materials.

\section{Water photoreduction}

In the context of water splitting with metal oxides, (including those with a complex stoichiometry, i.e. ternary oxides), reports on photocathodes are much scarcer than those dealing with photoanodes. In principle, metal oxide photocathodes are advantageous as compared to traditional III-V or II-VI semiconductors (e.g. GaAs or CdS) as they tend to be more stable in 
the dark, cost-effective, and available (usually made of common metals). ${ }^{25}$ However, promising p-type metal oxides generally have a tendency to photocorrode in aqueous environments, ${ }^{25}$ which limits their practical application. Therefore, significant efforts in the last years have been devoted to finding low band gap, stable and efficient metal oxide photocathodes, engineered with strategies such as nanostructuring, formation of heterojunctions, deposition of protective layers or loading of co-catalyts. ${ }^{111}$

Binary and ternary oxides. Among binary oxides, copper compounds have attracted most of the attention. Specifically, $\mathrm{Cu}_{2} \mathrm{O}$ displays the highest photoelectrochemical performance, ${ }^{112-115}$ as it has a rather narrow band gap, allowing for the absorption of most of the visible spectrum. However, it is difficult to achieve a reasonable material photostability. ${ }^{116,117}$ The most common approach adopted for protecting copper(I) oxide is the conformal deposition of a thin overlayer of $\mathrm{TiO}_{2}$ by ALD. ${ }^{112}$ The high cost and low scalability of this method precludes its widespread use for large size electrodes in practical devices. Recently in our laboratory, Cots et $a l^{117}$ synthesized $\mathrm{CuO}$ nanowire array electrodes with high photoactivity (Fig. 8a) and a faradaic efficiency for hydrogen generation of $45 \%$. To protect the photoelectrode against photocorrosion, an iron solution was drop-casted on the $\mathrm{CuO}$ electrodes, which upon a heat treatment lead to a $\mathrm{CuFe}_{2} \mathrm{O}_{4}$ spinel shell. Upon this treatment, although a decrease of the photocurrent (and the dark current) occurs (Fig. 8a and b), a significant stabilization of the photoresponse was observed (Fig. 8c), increasing the HER faradaic efficiency to $100 \%$. Thus, this method is beneficial for practical purposes due to its cost-effectiveness and potential scalability, although it needs to be effectively combined with co-catalysts to enhance the response.

On the other hand, ternary oxides comprise a wide number of promising materials, such as spinels, delafossites, perovskites, scheelites and other families, which could potentially fulfill the requirements for light absorption, stability and photoactivity for solar hydrogen generation. Among them, $\mathrm{CuBi}_{2} \mathrm{O}_{4}$ has attracted considerable interest, ${ }^{118-120}$ reaching relatively high photocurrents, although instability under illumination has also been reported. ${ }^{121}$

In this context, $\mathrm{CuFe}_{2} \mathrm{O}_{4}$ spinel stands out because of its non-toxicity, environmental benignity and its composition based on $\mathrm{Cu}$ and $\mathrm{Fe}$, which are earth-abundant elements. In this regard, we have prepared nanostructured spinel films by doctor blading a paste of commercial nanoparticles $(30 \mathrm{~nm}$ in diameter). ${ }^{122}$ The bare electrodes presented small anodic photocurrents. However, once subjected to an oxidative electrochemical treatment (i.e. multiple voltammetric cycles up to a sufficiently positive potential, concretely, $1.6 \mathrm{~V}_{\mathrm{RHE}}$ in basic media), the photoelectrode character shifted to p-type, with an enhanced photoresponse. We hypothesized that the electrochemical treatment induces the formation of acceptor species in the solid. This strategy could open up new prospects for the formation of self-dopant species within metal oxide structures. From a practical perspective, as the photocurrent onset potential of the modified electrode was located at $1.1 \mathrm{~V}_{\mathrm{RHE}}$, a
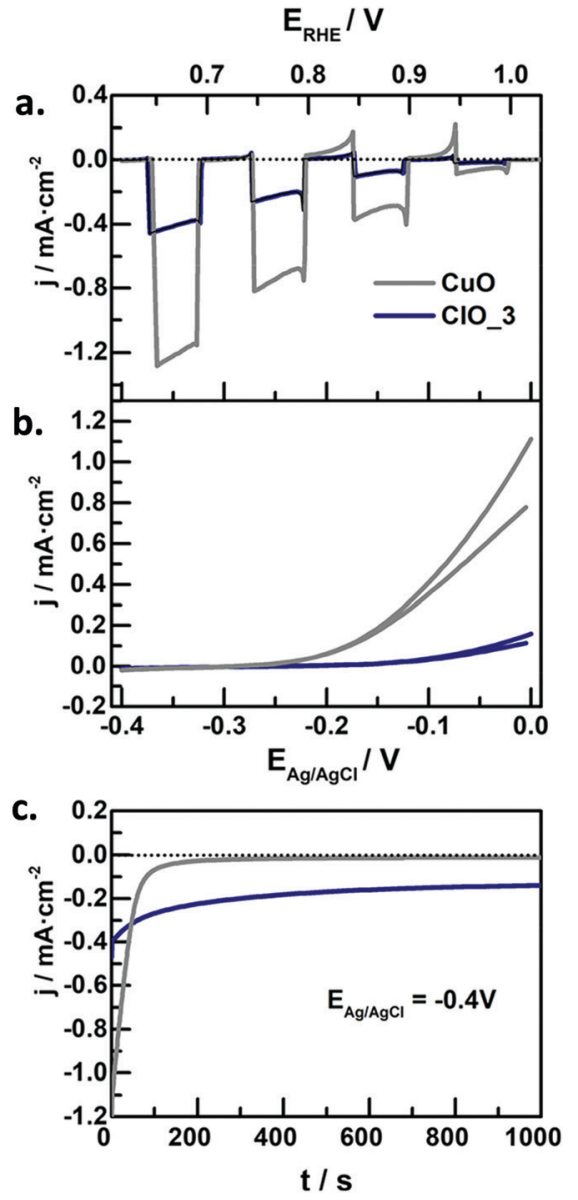

Fig. 8 (a) Linear sweep voltammetry under transient illumination, (b) cyclic voltammograms in the dark, and (c) photocurrent at $-0.4 \mathrm{~V}$ vs. $\mathrm{Ag} / \mathrm{AgCl}$ for $\mathrm{CuO}$ (grey lines) and Fe-modified $\mathrm{CuO}$ (blue lines) electrodes in $\mathrm{N}_{2}$-purged $1 \mathrm{M} \mathrm{NaOH}$. Illumination source: $320 \mathrm{~mW} \mathrm{~cm}^{-2}, \lambda>350 \mathrm{~nm}$. Reprinted with permission from ref. 117. Copyright 2018 American Chemical Society.

rather positive value, it is feasible to combine $\mathrm{CuFe}_{2} \mathrm{O}_{4}$ with an n-type material in a tandem PEC device. In a similar way, Park et al. ${ }^{123}$ developed a flame-annealing method to synthesize $\mathrm{CuFe}_{2} \mathrm{O}_{4}$ electrodes with a low density of oxygen vacancies, reaching photocurrents exceeding all the previously reported values. However, the stability issue still needs to be addressed for the practical use of this oxide.

Another interesting group of materials is constituted by $\mathrm{Cu}(\mathrm{I})$ delafossites, which have been studied as transparent conductive oxides (TCOs) for optoelectronic devices, ${ }^{124}$ as they generally exhibit high carrier mobility and transparency. Among the delafossite family, $\mathrm{CuFeO}_{2}$ should be highlighted owing to its narrow band gap $(1.5 \mathrm{eV})$, being the most studied delafossite in the context of water splitting. ${ }^{125-128}$ Despite its generally good stability in aqueous environments, the photocurrents in the absence of $\mathrm{O}_{2}$ (as electron scavenger) are very low. It has been reported that Fermi level pinning linked to the $\mathrm{Fe}^{3+} / \mathrm{Fe}^{2+}$ couple hinders the HER reaction in this material. ${ }^{129,130}$ Remarkably, NiFe an CoFe layered double hydroxides (LDHs) co-catalysts on $\mathrm{CuFeO}_{2}$ have led to major improvements in the 
photoresponse. ${ }^{127,128}$ Díaz-García et al. ${ }^{131}$ studied thin transparent $\mathrm{CuCrO}_{2}$ films as photocathodes for water splitting synthesized by a simple sol-gel method. In contrast with $\mathrm{CuFeO}_{2}$, the as-prepared bare electrodes already showed a relatively high IPCE $(6 \%$ at $350 \mathrm{~nm})$ for proton reduction with a rather positive photocurrent onset potential and remarkable photostability in both acidic and alkaline media. In view of the outstanding photoelectrochemical properties of this material, increasing its visible light absorption (for instance, by doping) seems imperative for its potential solar application, as the pristine material has a band gap of $3.15 \mathrm{eV}$.

Iron perovskites have been also investigated for water splitting, including $\mathrm{BiFeO}_{3},{ }^{132,133} \mathrm{YFeO}_{3},{ }^{134}$ and $\mathrm{LaFeO}_{3}$, being the latter the most studied. Lanthanum iron perovskite electrodes can exhibit both photoanodic or photocathodic behavior, depending on the synthetic route and conditions. ${ }^{135-138} \mathrm{We}$ have reported a straightforward wet chemical method to synthesize p-type thin film $\mathrm{LaFeO}_{3}$ electrodes. ${ }^{137}$ By introducing $\mathrm{Mg}$ or $\mathrm{Zn}$ as dopants, the p-type character is enhanced. For a $\mathrm{LaFe}_{0.95} \mathrm{M}_{0.05} \mathrm{O}_{3}(\mathrm{M}=\mathrm{Mg}$ or $\mathrm{Zn})$ stoichiometry, a 6-fold increase of the photocurrent is attained in the presence of $\mathrm{O}_{2}$ with respect to the pristine material (Fig. 9a and b). This effect is attributed to the substitution of trivalent Fe atoms by divalent $\mathrm{Mg}$ and $\mathrm{Zn}$ atoms in the perovskite lattice, which likely induces: (i) an increase of the $\mathrm{Fe}^{4+}$ density, and (ii) an improvement of carrier mobility due to lattice distortion. Impedance spectroscopy analysis through Mott-Schottky and Nyquist plots confirmed an increase in both charge carrier density and conductivity. Doping with equivalent quantities of either $\mathrm{Mg}$ or $\mathrm{Zn}$ led to similar photocurrent values, suggesting that both cations produce similar effects within the perovskite structure (Fig. 9).

Modeling impedance measurements with ternary oxides. Understanding the SEI under illumination is helpful to unveil the critical steps limiting the photoresponse of a particular material. In turn, this should facilitate the selection of appropriate strategies for improving their photoelectrochemical performance. Impedance spectroscopic techniques can provide important clues in this regard. The most commonly followed approach consists in modeling the interface as a combination of resistances and capacitors (equivalent circuits). ${ }^{139-141}$ In contrast, models comprising kinetic steps and the capacitive behavior of the double layer are significantly more complex, although the obtained model parameters have a clearer physical meaning.

Traditionally, these studies have focused on n-type oxide materials, but they can be easily extended to p-type SCs. For instance, Ponomarev et al. ${ }^{142}$ investigated $\mathrm{p}$-InP electrodes only by appropriate sign reversal of a model valid for n-type photoelectrodes. Later on, some of us $^{143}$ used the model developed by Leng et al. ${ }^{144}$ for $\mathrm{n}-\mathrm{TiO}_{2}$ to study $\mathrm{p}-\mathrm{CaFe}_{2} \mathrm{O}_{4}$ photocathodes (Fig. 10a). Electrons were considered as minority carriers, and the HER mechanism taken into account (Fig. 10b). For the first time, as far as we know, a kinetic model (without using equivalent circuits) was employed to describe the SEI at an illuminated metal oxide photocathode. A remarkable good fit of
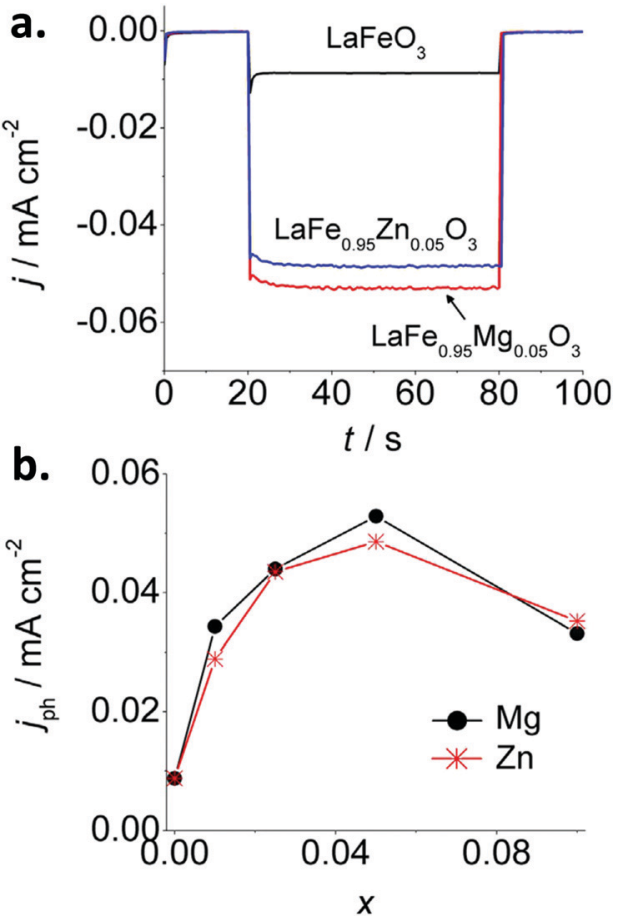

Fig. 9 (a) Photocurrent transients at $-0.25 \mathrm{~V}$ vs. $\mathrm{Ag} / \mathrm{AgCl}$ for pristine $\mathrm{LaFeO}_{3}$ and doped $\mathrm{LaFe}_{1-x} \mathrm{M}_{x} \mathrm{O}_{3}(\mathrm{M}=\mathrm{Mg}$ or $\mathrm{Zn}$ ) with $x=0.05$ and (b) stationary photocurrent at $-0.25 \mathrm{~V}$ vs. $\mathrm{Ag} / \mathrm{AgCl}$ after $60 \mathrm{~s}$ of illumination as a function of $x$ in $\mathrm{LaFe}_{1-x} \mathrm{M}_{x} \mathrm{O}_{3}\left(\mathrm{M}=\mathrm{Mg}\right.$ or $\mathrm{Zn}$ ) electrodes in $\mathrm{O}_{2}$-purged $0.1 \mathrm{M} \mathrm{NaOH}$. Reprinted with permission from ref. 137. Copyright 2017 Wiley-VCH Verlag GmbH \& Co.

the experimental data (in both Nyquist and Bode plots, Fig. 10c) was achieved. The following mechanism was assumed (Fig. 10b): (i) electrons in the conduction band can be trapped at surface states or be transferred to the electrolyte, (ii) recombination can take place in the bulk and via surface states, and (iii) electron transfer to the electrolyte likely occurs from the conduction band rather than from surface states. Both recombination and charge transfer kinetic constants were observed to depend on the applied potential, suggesting that an intermediate situation between Fermi level pinning and band edge pinning prevails at the SEI. In addition, it was also demonstrated that most of the photogenerated charge carriers recombine in the SC bulk.

\section{Tandem processes and modeling}

As discussed above, the fact that the oxides can be employed for both cathodic and anodic processes in artificial photosynthesis, including water photosplitting, makes feasible to devise all-oxide-based photosynthetic devices. Fig. 11 illustrates the three modalities in which artificial photosynthesis can occur in the case of systems formed by nanoparticles of oxides immersed in an aqueous medium. We have, on the one hand, the system consisting of a single semiconductor oxide (Fig. 11, left). It is very difficult to find a material that produces the photoelectrolysis of water with reasonable efficiency in this case, since it is necessary that: (i) both the CB and VB edges 

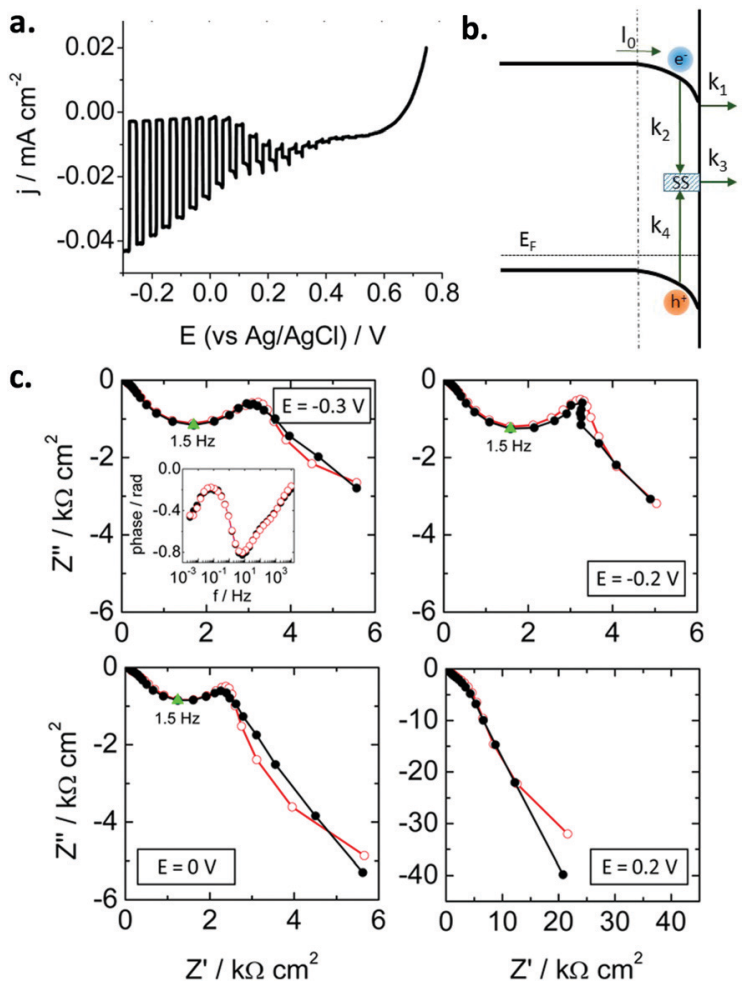

Fig. 10 (a) Linear sweep voltammogram under transient illumination for a calcium ferrite electrode. (b) Scheme illustrating the different charge transfer processes at the p-type semiconductor-electrolyte interface (SEI). (c) Nyquist plots under illumination for different potentials showing experimental points (black solid symbols) and simulated curves (red open symbols). Points corresponding to $\omega_{\max }$ are marked in green. Inset: Experimental and simulated Bode plots at $-0.3 \mathrm{~V}$. Illumination: $93 \mathrm{~mW}$ $\mathrm{cm}^{-2}$, AM 1.5G. Electrolyte: $\mathrm{N}_{2}$-purged $0.1 \mathrm{M} \mathrm{Na}_{2} \mathrm{SO}_{4}$. Reprinted with permission from ref. 143. Copyright 2016 American Chemical Society.

straddle the levels corresponding to the redox pairs $\mathrm{H}_{2} \mathrm{O} / \mathrm{H}_{2}$ and $\mathrm{O}_{2} / \mathrm{H}_{2} \mathrm{O}$, and (ii) the band gap is narrow enough as to enable efficient sunlight harvesting. In addition, it should be noted that there must be some extra driving force for the transfer of charge carriers to occur (because of the existence of significant overpotentials, particularly for water oxidation). In contrast, the realization of artificial photosynthesis in two stages makes it possible to obtain greater efficiencies and multiplies the possibility of finding more promising systems since two photoactive materials are now combined. In this case, oxidation and reduction photoreactions occur in separate materials whose por n-type characteristics and the position of their bands have been optimized to perform one of the half-reactions. In addition, having the two redox processes spatially separated enables the search of specific co-catalysts for both anodic and cathodic reactions.

As observed, in the case of systems consisting of dispersed semiconductor particles, there are two possibilities to combine the two photoactive materials. First, a reversible redox pair whose level must be between the $\mathrm{CB}$ edge of the material that supports photooxidation and the $\mathrm{VB}$ edge of the material supporting photoreduction (Fig. 11, center) can be employed. Secondly, the direct particle junction of the two photoactive materials could be envisaged (Fig. 11, right). Among the oxides commonly employed for water oxidation, one finds $\mathrm{TiO}_{2}, \mathrm{WO}_{3}$, $\mathrm{Fe}_{2} \mathrm{O}_{3}, \mathrm{BiVO}_{4}, \mathrm{ZnO}, \mathrm{CoO}_{x}, \mathrm{CoTiO}_{3}, \mathrm{LaFeO}_{3}, \mathrm{BiO}_{x}$ and oxides with more complex stoichiometries based on $\mathrm{Bi}$ and either $\mathrm{Ta}$ or $\mathrm{Nb}$. The choice of oxides for sustaining water reduction is much more limited, being $\mathrm{SrTiO}_{3}$ with an appropriate co-catalyst, the most typical. ${ }^{145,146}$

The concepts presented above for a dispersed particle system can be easily extended to photoelectrochemical cells. Fig. 12 illustrates the functioning of photoelectrolysis cells based on a photoanode + dark cathode (Fig. 12, one stage left), a photocathode + dark anode (Fig. 12, one stage right), and a photoanode + photocathode combination (Fig. 12, left). The latter are made up of two photoelectrodes: one n-type, which works as a photoanode and another p-type, which acts as a photocathode. This is the way in which the so-called tandem cells are constructed. In tandem cells, both electrodes are photoexcited by the same light beam. This is achieved with photoelectrodes whose light absorption is complementary. The light passes first through the wide band gap material and

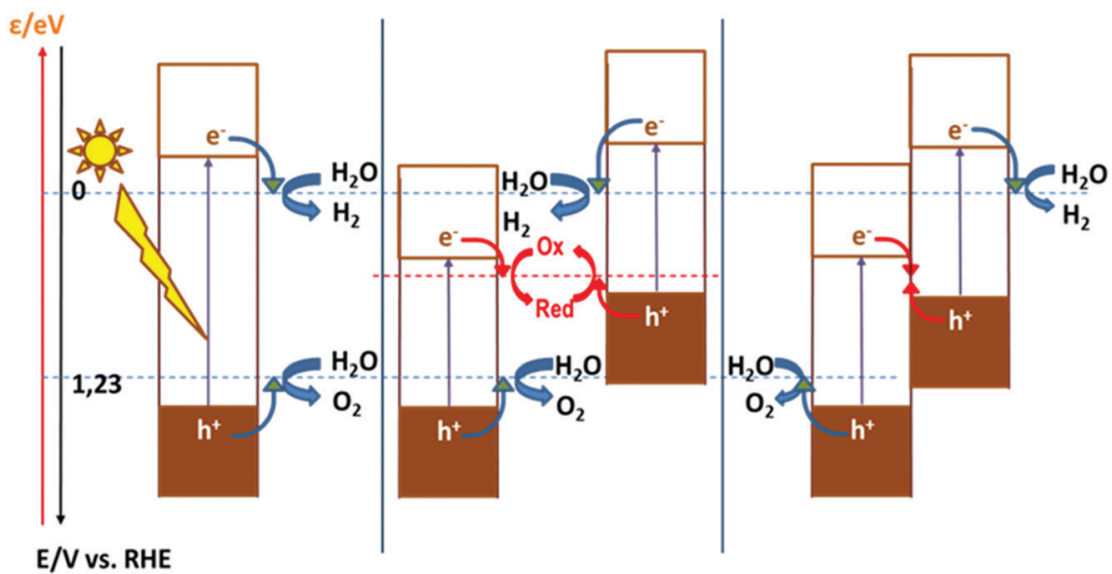

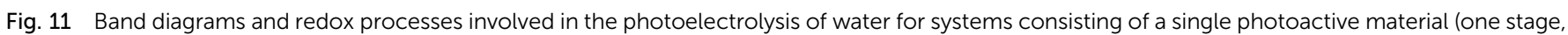
left) and two photoactive materials in the presence of a redox mediator (center) or by direct contact (right). 


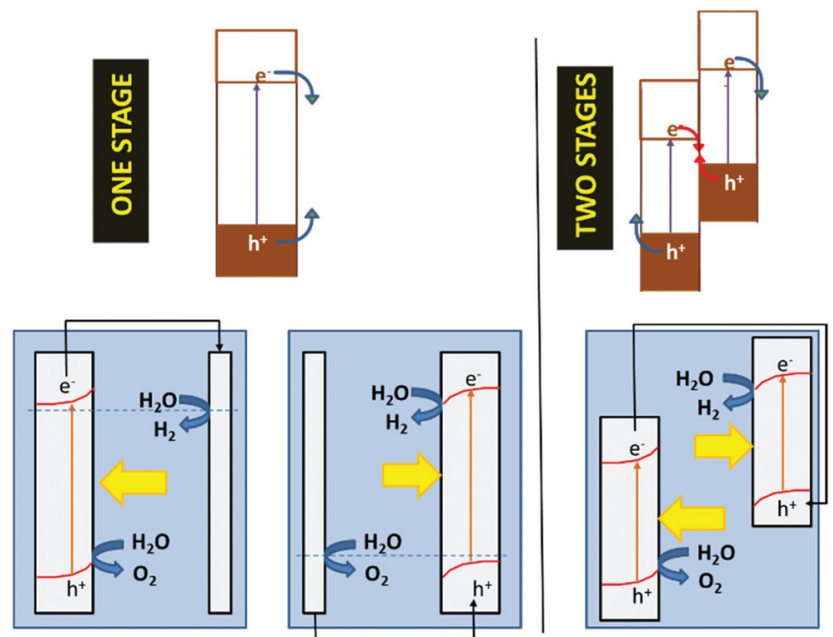

Fig. 12 A schematic illustrating the photoelectrochemical variants of artificial photosynthesis systems in one and two stages. In the case of the one-stage system, the photoelectrode could sustain either the oxidation (photoanode) or reduction (photocathode) process, while the other half-reaction is performed by a conventional non-photoactive electrode (cathode or anode, respectively). In the case of the two-stage system, where a photoanode and a photocathode are connected in series, the electrochemical device is known as a tandem cell.

subsequently, it is absorbed by the narrow band gap material. Obviously, the electrolyte solution separating the electrodes could absorb part of the light, causing efficiency to decrease. One could conceive photoelectrochemical cells with two photoelectrodes that are illuminated in parallel rather than in series. These are also considered by many authors as tandem cells. ${ }^{147}$

It is well-known that developing tandem cells for solar fuel generation may benefit from the facts that: (i) it is more likely to find a pair of photoelectrodes whose band edges are located at the appropriate energies to carry out water photooxidation and water or $\mathrm{CO}_{2}$ photoreduction than to identify a single photoactive material; (ii) it is easier to find specific co-catalysts for each of the redox processes so that there is no interference between them; and (iii) the maximum theoretical efficiency that can be achieved is larger than in cells with a single photoelectrode. ${ }^{148,149}$

Lately, we have been devoting a significant effort for the clarification of the factors that influence the solar-to-hydrogen (STH) conversion efficiency either under zero-bias conditions or under applied bias (Applied bias photo-to-current efficiency, $\mathrm{ABPE}$, for hydrogen generation). Although this question has been dealt with in several papers, a quasi-analytical approach based on the classical Gärtner model has not been reported yet.

We have recently embarked on such a project. It is instructive to show that the ABPE (or the STH efficiency) may substantially differ from the maximum value calculated on the basis of electrode band gaps because of different properties that are central in the field, such as, for instance, the diffusion length of the minority carrier, the separation of photocathode and photoanode flat band potentials, the absorption coefficients, or the doping density. The electronic structure of oxides is characterized by a weak curvature of the energy $v s$. momentum curve, leading to large effective masses for the charge carriers and thus, to short diffusions lengths. This is particularly critical for the minority carriers. Thus, efficiency calculations are pertinent as the photoelectrochemical behavior is most of the times far away from ideal, limiting the usefulness of typical theoretical studies. It is worth noting that recent studies have attempted to address this question, at least partially. ${ }^{149,150}$ In our model we have neglected mass transport limitations and light absorption by the electrolyte, while the behavior of both electrodes has been considered to follow the ideas delineated by Gärtner, either for front-side or back-side illumination. Light absorption is considered to follow a simple model (Tauc relationship). In addition, surface recombination and carrier transfer constants are considered in an explicit way.

To illustrate the potential usefulness of this type of calculations, Fig. 13 shows a set of ABPE curves as a function of the applied bias (V) for different values of the doping density (Fig. 13a), minority carrier diffusion length (Fig. 13b), absorption coefficients (Fig. 13c) and difference between flat band potentials of photocathode and photoanode (Fig. 13d). All these properties refer to the photoanode (wide band gap material). As observed, relatively high values for the conversion efficiency can be attained for the reference scenario with substantial surface recombination and a fixed photocathode. The effect of the parameters mentioned above on the maximum ABPE is dramatic, particularly in the case of the absorption coefficient and the minority carrier diffusion length. In any case, this type of calculations should be useful for guiding the choice of the most promising materials taking into account the intrinsic non-ideality of practical oxide electrodes.

Together with the problem of low efficiencies, what makes it difficult to develop devices is to find semiconductor electrode materials presenting enough stability in contact with the typical either acid or alkaline aqueous media. Oxides will tend to be more stable in an aqueous environment than semiconductors such as chalcogenides. In fact, some photoanode materials have enough stability in acid (e.g. $\left.\mathrm{WO}_{3}\right)$ or alkaline (e.g. $\mathrm{Fe}_{2} \mathrm{O}_{3}$ ) environments. However, the challenge of finding efficient and stable p-type oxide electrodes still needs to be addressed.

A direction to palliate the stability problem could be based on alternative photoelectrolyzer designs. In this respect, it is particularly interesting to advance toward the development of quasi-solid-sate devices in which the liquid electrolyte is substituted by a polymer electrolyte membrane. Recent results obtained in our laboratory in collaboration with Aricò's laboratory (ITAE-CNR, Italy) show that this option is feasible by working with a tandem device based on a hematite photoanode and a $\mathrm{CuO}$ photocathode. Photoresponses like those obtained in a conventional liquid electrolyte were obtained, but with a greater stability of the semiconductor electrodes. ${ }^{151,152}$ This is particularly important in the case of the photocathode, which is prone to severe photocorrosion, except for some delafossites, as mentioned above. ${ }^{126,129,131}$

Although significant progress has been made in the last years, the development of practical water splitting (artificial 

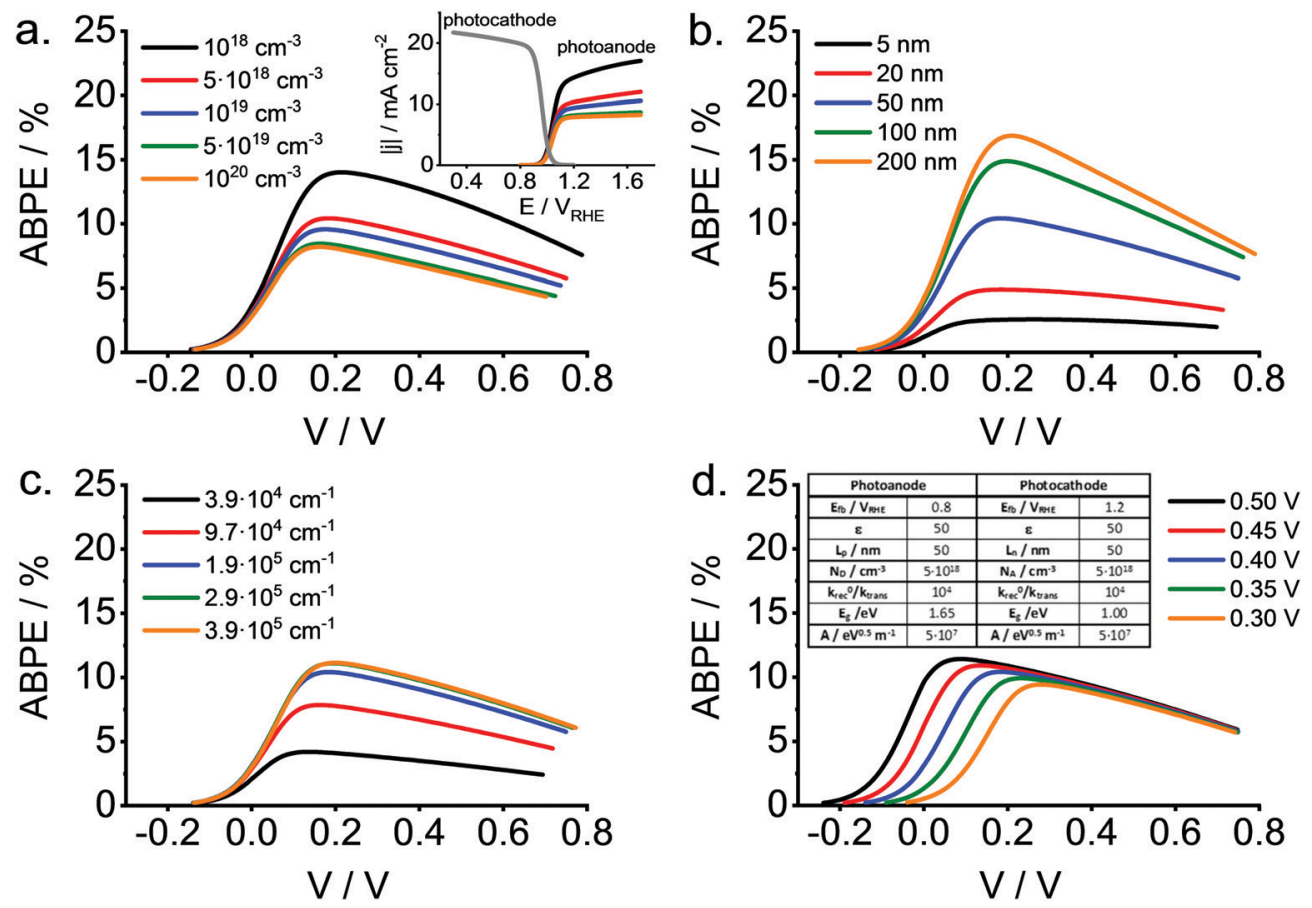

Fig. 13 ABPE vs. bias $(V)$ curves of a tandem cell for different values of the photoanode properties, including: (a) doping density, $N_{D}$; (b) minority carrier diffusion length, $L_{p}$; (c) absorption coefficient values at $400 \mathrm{~nm}$ (assuming direct band gap and Tauc equation: $\alpha h \nu=A\left(h \nu-E_{\mathrm{g}}\right)^{0.5}$, for different values of $A$ ranging from $10^{7}$ to $10^{8} \mathrm{eV}^{0.5} \mathrm{~m}^{-1}$ ); and (d) difference between the flat band potential for the photocathode and the photoanode. Inset in (d) shows the model parameters used in the calculation, being the photoanode thickness of $100 \mathrm{~nm}$. Inset in (a) displays photocurrent (absolute values) as a function of potential for both photocathode and photoanode (for different doping densities).

photosynthesis) photoelectrochemical devices requires additional effort. Both the conversion efficiency and the stability of the photoelectrodes need to be addressed, considering that they scale differently with the band gap. Increasing the conversion efficiency will depend on finding oxides with narrow band gap and appropriately engineered bulk and surface properties promoting carrier mobility and transfer to the electrolyte. For increasing stability, three compatible directions could be explored: (i) finding new materials (ternary or multinary oxides); (ii) identifying appropriate means of protecting the electrode surface, including the use of co-catalysts; and (iii) substituting the conventional liquid electrolytes by polymer electrolytes. The latter will bring about new device designs closer to the flat configuration desirable for the mass deployment of this technology.

\section{Conclusions and outlook}

In recent years there has been a tremendous effort to develop new electrode materials based on transition metal oxides. However, there are many aspects that still need to be improved to enable their use in devices converting solar energy into chemical energy or in environmental applications. The Achilles' heels of these materials are their sometimes low stability in contact with aqueous media under illumination as well as their low conversion efficiency.

The stability of oxides is generally better than that of other semiconductors (e.g. chalcogenides), and some of them show a satisfactory photostability for many applications (e.g. titanium dioxide, tungsten trioxide and hematite); however, durability should be improved in the cost-effective copper-based binary and ternary oxides, which commonly have a clear tendency to photocorrode. Thus, work to stabilize these materials is still needed. In relation to the low conversion efficiency, methods that modify both the bulk and the surface of the semiconductor electrodes should be considered, facilitating the mobility of carriers and decreasing recombination both in the bulk and on the surface. It is worth noting that a simple strategy to improve both stability and conversion efficiency is based on nanostructuring the electrode materials. The preparation of structures ordered at the nanoscopic level with quasi-one-dimensional objects (e.g. nanocolumns, nanorods or nanofibers) is especially interesting, provided the nanoobject diameter can be tuned to make compatible an extended interfacial area with the existence of a space charge region in the electrodes, which certainly enhances charge separation and decreases recombination within the semiconductor.

The experimental work required to achieve efficient and stable oxide electrodes should be guided (both in their a priori orientation and a posteriori rationalization) by theoretical physicochemical methods for, among others: (i) the selection of new oxide materials as potential candidates in the photoelectrochemical processes of interest; (ii) the extraction of microscopic information on the kinetics of the different electrode processes occurring under illumination; (iii) the interpretation of the experimental results with kinetic models; and (iv) the 
development of suitable devices to carry out artificial photosynthesis. Regarding the materials, there are many ternary and multinary metal oxides that are yet to be explored; the enormous number of candidates advises the use of DFT calculations to filter them down to an initial selection that serves as a starting point for further experimental work. Regarding the devices, models should comprise transport phenomena (both in the electrolyte and the semiconductor), besides the material and kinetic aspects of the photoinduced charge transfer phenomena.

Finally, as a prospective outlook for the future research with metal oxides as photoelectrocatalysts, we propose here a succinct guideline to each of the issues mentioned above. Regarding materials stability, the use of protective layers (made of materials fully stable in aqueous media) should be explored in the future, but with greater emphasis on cost efficiency and scalability than up to now; moreover, the use of co-catalysts that channel the transfer to solution of minority carriers is a method of improving stability and additional efforts should be made in this direction. As for their low efficiency, protective layers can promote increased conversion efficiency by allowing selective transport of minority carriers, while blocking that of majority carriers. In any case, the use of co-catalysts and doping will be of particular importance.

Concerning the modeling of materials, DFT calculations will provide reliable electronic structures for the oxides. We expect that, in the coming years, the number and quality of publications on oxide electronic structures and on the electronic and optical properties derived therefrom will increase. This approach will also be useful for optimizing photoactivity improvement strategies, such as doping. On the other hand, kinetic modeling should allow the identification of the limiting steps under operation and it is therefore especially useful to unravel all the information provided by the electrochemical methods resolved in the frequency or time domains. Finally, at the device level, the theoretical studies should guide the cell design, having in mind the potential up-scaling and marketing, while helping to improve the conversion efficiency and especially the stability of the photoelectrodes. In this sense, the development of tandem photoelectrochemical cells based on polymeric electrolyte membranes can be a pathway for designing durable and scalable devices.

All in all, we believe that in the near future new opportunities will continue to appear for researchers who are currently working on these issues as well as for newcomers wanting to join the exciting challenge of achieving conversion and storage of solar energy in a cost-effective and environmentally benign way. There is no doubt that in the coming years, efforts will concentrate in this direction, to contribute to the decarbonization of our energy system and our economy. The multidisciplinary approach that we have illustrated in this contribution can hopefully serve as an inspiration for the emergence of new and promising ideas.

\section{Conflicts of interest}

There are no conflicts to declare.

\section{Acknowledgements}

Continued support from the Spanish Ministry of Science and Innovation (MICINN) is gratefully acknowledged, in particular through the current project RTI2018-102061-B-I00 (FONDOS FEDER). Financial support from the Generalitat Valenciana through project PROMETEO/2020/089 is also thanked. Finally, we are also grateful to our many collaborators during the last 15 years, who also made this contribution possible.

\section{Notes and references}

1 N. S. Lewis and D. G. Nocera, Proc. Natl. Acad. Sci. U. S. A., 2006, 103, 15729-15735.

2 D. G. Nocera, Acc. Chem. Res., 2017, 50, 616-619.

3 A. J. Bard, J. Photochem., 1979, 10, 59-75.

4 K. Rajeshwar, A. Thomas and C. Janaky, J. Phys. Chem. Lett., 2015, 6, 139-147.

5 M. Anne Fox and A. J. Bard, Acc. Chem. Res., 1995, 28, 141-145.

6 F. E. Osterloh, ACS Energy Lett., 2017, 2, 445-453.

7 K. Rajeshwar, J. Phys. Chem. Lett., 2011, 2, 1301-1309.

8 H. Kisch, Angew. Chem., Int. Ed., 2013, 52, 812-847.

9 S. E. Braslavsky, A. M. Braun, A. E. Cassano, A. V. Emeline, M. I. Litter, L. Palmisano, V. N. Parmon and N. Serpone, Pure Appl. Chem., 2011, 83, 931-1014.

10 H. Park, H. Il Kim, G. H. Moon and W. Choi, Energy Environ. Sci., 2016, 9, 411-433.

11 B. Ohtani, J. Photochem. Photobiol., C, 2010, 11, 157-178.

12 B. Ohtani, Phys. Chem. Chem. Phys., 2014, 16, 1788-1797.

13 J. Yang, D. Wang, H. Han and C. Li, Acc. Chem. Res., 2013, 46, 1900-1909.

14 P. Lianos, Appl. Catal., B, 2017, 210, 235-254.

15 K. Sivula and R. Van De Krol, Nat. Rev. Mater., 2016, 1, 15010.

16 A. Hankin, F. E. Bedoya-Lora, C. K. Ong, J. C. Alexander, F. Petter and G. H. Kelsall, Energy Environ. Sci., 2017, 10, 346-360.

17 J. Li and N. Wu, Catal. Sci. Technol., 2015, 5, 1360-1384.

18 Y. Yang, S. Niu, D. Han, T. Liu, G. Wang and Y. Li, Adv. Energy Mater., 2017, 7, 1-26.

19 C. Jiang, S. J. A. Moniz, A. Wang, T. Zhang and J. Tang, Chem. Soc. Rev., 2017, 46, 4645-4660.

20 K. Sivula, J. Phys. Chem. Lett., 2013, 4, 1624-1633.

21 S. Jung, C. C. L. McCrory, I. M. Ferrer, J. C. Peters and T. F. Jaramillo, J. Mater. Chem. A, 2016, 4, 3068-3076.

22 F. Song, L. Bai, A. Moysiadou, S. Lee, C. Hu, L. Liardet and X. Hu, J. Am. Chem. Soc., 2018, 140, 7748-7759.

23 F. E. Osterloh, Chem. Soc. Rev., 2013, 42, 2294-2320.

24 S. Chen, S. S. Thind and A. Chen, Electrochem. Commun., 2016, 63, 10-17.

25 I. Sullivan, B. Zoellner and P. A. Maggard, Chem. Mater., 2016, 28, 5999-6016.

26 N. K. Awad, E. A. Ashour and N. K. Allam, J. Renewable Sustainable Energy, 2014, 6, 022702.

27 Z. Haider, H. W. Yim, H. W. Lee and H. il Kim, Int. J. Hydrogen Energy, 2020, 45, 5793-5815.

28 Z. Chen, T. F. Jaramillo, T. G. Deutsch, A. Kleiman-Shwarsctein, A. J. Forman, N. Gaillard, R. Garland, K. Takanabe, C. Heske, M. Sunkara, E. W. McFarland, K. Domen, E. L. Milled and H. N. Dinh, J. Mater. Res., 2010, 25, 3-16.

29 H. Dotan, K. Sivula, M. Grätzel, A. Rothschild and S. C. Warren, Energy Environ. Sci., 2011, 4, 958-964.

30 X. Zhao, W. Luo, J. Feng, M. Li, Z. Li, T. Yu and Z. Zou, Adv. Energy Mater., 2014, 4, 1-7.

31 N. Guijarro, M. S. Prévot and K. Sivula, Phys. Chem. Chem. Phys., 2015, 17, 15655-15674.

32 T. Berger, D. Monllor-Satoca, M. Jankulovska, T. Lana-Villarreal and R. Gómez, ChemPhysChem, 2012, 13, 2824-2875.

33 S. Hennessey and P. Farràs, Chem. Commun., 2018, 54, 6662-6680.

34 L. M. Peter, Electroanalysis, 2015, 27, 864-871.

35 C. Janáky and K. Rajeshwar, ACS Energy Lett., 2017, 2, 1425-1428.

36 W. J. Yin, H. Tang, S. H. Wei, M. M. Al-Jassim, J. Turner and Y. Yan, Phys. Rev. B: Condens. Matter Mater. Phys., 2010, 82, 1-6. 
37 M. Woodhouse and B. A. Parkinson, Chem. Soc. Rev., 2009, 38, 197-210.

38 H. Gerischer, J. Electroanal. Chem., 1975, 58, 263-274.

39 T. Lana Villarreal, R. Gómez, M. Neumann-Spallart, N. AlonsoVante and P. Salvador, J. Phys. Chem. B, 2004, 108, 15172-15181.

40 W. W. Gärtner, Phys. Rev., 1959, 116, 84-87.

41 A. Hagfeldt, H. Lindström, S. Södergren and S. E. Lindquist, J. Electroanal. Chem., 1995, 381, 39-46.

42 P. V. Kamat, J. Phys. Chem. Lett., 2012, 3, 663-672.

43 P. V. Kamat, Acc. Chem. Res., 2017, 50, 527-531.

44 A. R. C. Bredar, A. L. Chown, A. R. Burton and B. H. Farnum, ACS Appl. Energy Mater., 2020, 3, 66-98.

45 F. Fabregat-Santiago, G. Garcia-Belmonte, J. Bisquert, A. Zaban and P. Salvador, J. Phys. Chem. B, 2002, 106, 334-339.

46 F. Fabregat-Santiago, I. Mora-Seró, G. Garcia-Belmonte and J. Bisquert, J. Phys. Chem. B, 2003, 107, 758-768.

47 Y. K. Gaudy and S. Haussener, J. Mater. Chem. A, 2016, 4, 3100-3114.

48 T. Shinagawa, Z. Cao, L. Cavallo and K. Takanabe, J. Energy Chem., 2017, 26, 259-269.

49 S. Hu, C. Xiang, S. Haussener, A. D. Berger and N. S. Lewis, Energy Environ. Sci., 2013, 6, 2984-2993.

50 P. C. K. Vesborg and B. Seger, Chem. Mater., 2016, 28, 8844-8850.

51 A. Solbrand, H. Lindström, H. Rensmo, A. Hagfeldt, S. E. Lindquist and S. Södergren, J. Phys. Chem. B, 1997, 101, 2514-2518.

52 A. Solbrand, A. Henningsson, S. Södergren, H. Lindström, A. Hagfeldt and S. E. Lindquist, J. Phys. Chem. B, 1999, 103, 1078-1083.

53 Y. Pleskov, Semiconductor Photoelectrochemistry, Springer, US, 1986.

54 N. Kopidakis, E. A. Schiff, N. G. Park, J. Van De Lagemaat and A. J. Frank, J. Phys. Chem. B, 2000, 104, 3930-3936.

55 T. Lana-Villarreal and R. Gómez, Chem. Phys. Lett., 2005, 414, 489-494.

56 M. Jankulovska, T. Berger, T. Lana-Villarreal and R. Gómez, Electrochim. Acta, 2012, 62, 172-180.

57 A. Cots, D. Cibrev, P. Bonete and R. Gómez, ChemElectroChem, 2017, 4, 585-593.

58 A. Hankin, F. E. Bedoya-Lora, J. C. Alexander, A. Regoutz and G. H. Kelsall, J. Mater. Chem. A, 2019, 7, 26162-26176.

59 J. Bisquert, Phys. Chem. Chem. Phys., 2003, 5, 5360-5364.

60 I. Abayev, A. Zaban, V. G. Kytin, A. A. Danilin, G. Garcia-Belmonte and J. Bisquert, J. Solid State Electrochem., 2007, 11, 647-653.

61 J. Bisquert, Phys. Chem. Chem. Phys., 2008, 10, 49-72.

62 J. Bisquert, F. Fabregat-Santiago, I. Mora-Seró, G. Garcia-Belmonte, E. M. Barea and E. Palomares, Inorg. Chim. Acta, 2008, 361, 684-698.

63 J. Bisquert and V. S. Vikhrenko, J. Phys. Chem. B, 2004, 108, 2313-2322.

64 H. Wang, J. He, G. Boschloo, H. Lindström, A. Hagfeldt and S. E. Lindquist, J. Phys. Chem. B, 2001, 105, 2529-2533.

65 N. Kopidakis, N. R. Neale, K. Zhu, J. Van De Lagemaat and A. J. Frank, Appl. Phys. Lett., 2005, 87, 1-3.

66 L. De La Garza, Z. V. Saponjic, N. M. Dimitrijevic, M. C. Thurnauer and T. Rajh, J. Phys. Chem. B, 2006, 110, 680-686.

67 T. Berger, T. Lana-Villarreal, D. Monllor-Satoca and R. Gómez, Electrochem. Commun., 2006, 8, 1713-1718.

68 T. Berger, T. Lana-Villarreal, D. Monllor-Satoca and R. Gómez, J. Phys. Chem. C, 2007, 111, 9936-9942.

69 T. Berger, T. Lana-Villarreal, D. Monllor-Satoca and R. Gómez, Chem. Phys. Lett., 2007, 447, 91-95.

70 M. Jankulovska, T. Berger, S. S. Wong, R. Gómez and T. LanaVillarreal, ChemPhysChem, 2012, 13, 3008-3017.

71 T. Lana-Villarreal, Y. Mao, S. S. Wong and R. Gómez, Nanoscale, 2010, 2, 1690-1698.

72 T. Berger, T. Lana-Villarreal, D. Monllor-Satoca and R. Gómez, J. Phys. Chem. C, 2008, 112, 15920-15928.

73 D. Cibrev, M. Tallarida, C. Das, T. Lana-Villarreal, D. Schmeisser and R. Gómez, Phys. Chem. Chem. Phys., 2017, 19, 21807-21817.

74 N. Guijarro, T. Lana-Villarreal and R. Gómez, Chem. Commun., 2012, 48, 7681-7683.

75 D. Monllor-Satoca and R. Gómez, J. Phys. Chem. C, 2008, 112, 139-147.

76 D. Cibrev, M. Jankulovska, T. Lana-Villarreal and R. Gómez, ACS Appl. Mater. Interfaces, 2014, 6, 10304-10312.
77 I. Mora-Seró, T. L. Villarreal, J. Bisquert, Á. Pitarch, R. Gómez and P. Salvador, J. Phys. Chem. B, 2005, 109, 3371-3380.

78 T. Lana-Villarreal, J. M. Pérez and R. Gómez, Surf. Sci., 2004, 572, 329-336.

79 T. Lana-Villarreal, A. Rodes, J. M. Pérez and R. Gómez, J. Am. Chem. Soc., 2005, 127, 12601-12611.

80 T. Lana-Villarreal, J. M. Pérez and R. Gómez, C. R. Chim., 2006, 9, 806-816.

81 T. Berger, J. M. Delgado, T. Lana-Villarreal, A. Rodes and R. Gómez, Langmuir, 2008, 24, 14035-14041.

82 T. Berger, A. Rodes and R. Gómez, Phys. Chem. Chem. Phys., 2010, 12, 10503-10511.

83 T. Berger, A. Rodes and R. Gómez, Chem. Commun., 2010, 46, 2992-2994.

84 N. E. Mendieta-Reyes, A. K. Díaz-García and R. Gómez, ACS Catal., 2018, 8, 1903-1912.

85 N. E. Mendieta-Reyes, W. Cheuquepán, A. Rodes and R. Gómez, ACS Catal., 2020, 10, 103-113.

86 A. Fujishima and K. Honda, Nature, 1972, 238, 37-38.

87 M. Grätzel, Nature, 2001, 414, 338-344.

88 D. Monllor-Satoca, T. Lana-Villarreal and R. Gómez, Langmuir, 2011, 27, 15312-15321.

89 H. Il Kim, G. H. Moon, D. Monllor-Satoca, Y. Park and W. Choi, J. Phys. Chem. C, 2012, 116, 1535-1543.

90 H. Il Kim, D. Monllor-Satoca, W. Kim and W. Choi, Energy Environ. Sci., 2015, 8, 247-257.

91 D. Monllor-Satoca, M. Bärtsch, C. Fàbrega, A. Genc, S. Reinhard, T. Andreu, J. Arbiol, M. Niederberger and J. R. Morante, Energy Environ. Sci., 2015, 8, 3242-3254.

92 A. K. Díaz-García and R. Gómez, J. Photochem. Photobiol., A, 2018, 366, 65-71.

93 W. Kim, T. Tachikawa, D. Monllor-Satoca, H. Il Kim, T. Majima and W. Choi, Energy Environ. Sci., 2013, 6, 3732-3739.

94 C. Fàbrega, S. Murcia-López, D. Monllor-Satoca, J. D. Prades, M. D. Hernández-Alonso, G. Penelas, J. R. Morante and T. Andreu, Appl. Catal., B, 2016, 189, 133-140.

95 T. H. Jeon, D. Monllor-Satoca, G.-H. Moon, W. Kim, H.-I. Kim, D. W. Bahnemann, H. Park and W. Choi, Nat. Commun., 2020, 11, 967.

96 M. Tallarida, C. Das, D. Cibrev, K. Kukli, A. Tamm, M. Ritala, T. Lana-Villarreal, R. Gómez, M. Leskelä and D. Schmeisser, J. Phys. Chem. Lett., 2014, 5, 3582-3587.

97 A. Cots, D. Cibrev, P. Bonete and R. Gómez, J. Solid State Electrochem., 2018, 22, 149-156.

98 A. Cots and R. Gómez, Appl. Catal., B, 2017, 219, 492-500.

99 D. Cibrev, M. Jankulovska, T. Lana-Villarreal and R. Gómez, Int. J. Hydrogen Energy, 2013, 38, 2746-2753.

$100 \mathrm{~J}$. Quiñonero, T. Lana-Villarreal and R. Gómez, Appl. Catal., B, 2016, 194, 141-149.

101 J. Quiñonero and R. Gómez, Appl. Catal., B, 2017, 217, 437-447.

102 S. Murcia-López, C. Fàbrega, D. Monllor-Satoca, M. D. HernándezAlonso, G. Penelas-Pérez, A. Morata, J. R. Morante and T. Andreu, ACS Appl. Mater. Interfaces, 2016, 8, 4076-4085.

103 D. Recatalá, R. Llusar, A. L. Gushchin, E. A. Kozlova, Y. A. Laricheva, P. A. Abramov, M. N. Sokolov, R. Gómez and T. Lana-Villarreal, ChemSusChem, 2015, 8, 148-157.

104 R. Franking, L. Li, M. A. Lukowski, F. Meng, Y. Tan, R. J. Hamers and S. Jin, Energy Environ. Sci., 2013, 6, 500-512.

105 Y. Liang, T. Tsubota, L. P. A. Mooij and R. Van De Krol, J. Phys. Chem. C, 2011, 115, 17594-17598.

106 Y. Park, K. J. Mc Donald and K. S. Choi, Chem. Soc. Rev., 2013, 42, 2321-2337.

107 H. Park and W. Choi, J. Phys. Chem. B, 2004, 108, 4086-4093.

108 J. Kim, D. Monllor-Satoca and W. Choi, Energy Environ. Sci., 2012, $5,7647-7656$.

109 D. Monllor-Satoca, R. Gómez, M. González-Hidalgo and P. Salvador, Catal. Today, 2007, 129, 247-255.

110 J. Quiñonero and R. Gómez, Electrochim. Acta, 2018, 274, 224-232.

111 W. Yang and J. Moon, ChemSusChem, 2019, 11, 1889.

112 A. Paracchino, V. Laporte, K. Sivula, M. Grätzel and E. Thimsen, Nat. Mater., 2011, 10, 456-461.

113 S. D. Tilley, M. Schreier, J. Azevedo, M. Stefik and M. Grätzel, Adv. Funct. Mater., 2014, 24, 303-311.

114 W. Niu, L. Zhu, Y. Wang, Z. Lou and Z. Ye, Catal. Sci. Technol., 2017, 7, 1602-1610. 
115 L. Pan, J. H. Kim, M. T. Mayer, M. K. Son, A. Ummadisingu, J. S. Lee, A. Hagfeldt, J. Luo and M. Grätzel, Nat. Catal., 2018, 1, 412-420.

116 W. Septina, R. R. Prabhakar, R. Wick, T. Moehl and S. D. Tilley, Chem. Mater., 2017, 29, 1735-1743.

117 A. Cots, P. Bonete and R. Gómez, ACS Appl. Mater. Interfaces, 2018, 10, 26348-26356.

118 D. Kang, J. C. Hill, Y. Park and K.-S. Choi, Chem. Mater., 2016, 28, 4331-4340.

119 F. Wang, W. Septina, A. Chemseddine, F. F. Abdi, D. Friedrich, P. Bogdanoff, R. van de Krol, S. D. Tilley and S. P. Berglund, J. Am. Chem. Soc., 2017, 139, 15094-15103.

120 I. Rodríguez-Gutiérrez, R. García-Rodríguez, M. Rodríguez-Pérez, A. Vega-Poot, G. Rodríguez Gattorno, B. A. Parkinson and G. Oskam, J. Phys. Chem. C, 2018, 122, 27169-27179.

121 S. P. Berglund, F. F. Abdi, P. Bogdanoff, A. Chemseddine, D. Friedrich and R. van de Krol, Chem. Mater., 2016, 28, 4231-4242.

122 M. I. Díez-García, T. Lana-Villarreal and R. Gómez, ChemSusChem, 2016, 9, 1504-1512.

123 S. Park, J. H. Baek, L. Zhang, J. M. Lee, K. H. Stone, I. S. Cho, J. Guo, H. S. Jung and X. Zheng, ACS Sustainable Chem. Eng., 2019, 7, 5867-5874.

124 F. F. Abdi and S. P. Berglund, J. Phys. D: Appl. Phys., 2017, 50, 193002

125 C. G. Read, Y. Park and K.-S. Choi, J. Phys. Chem. Lett., 2012, 3, 1872-1876.

126 M. S. Prévot, N. Guijarro and K. Sivula, ChemSusChem, 2015, 8, 1359-1367.

127 Y. J. Jang, Y. Bin Park, H. E. Kim, Y. H. Choi, S. H. Choi and J. S. Lee, Chem. Mater., 2016, 28, 6054-6061.

128 Y. Oh, W. Yang, J. Tan, H. Lee, J. Park and J. Moon, Adv. Funct. Mater., 2019, 29, 1-10.

129 M. S. Prévot, X. A. Jeanbourquin, W. S. Bourée, F. Abdi, D. Friedrich, R. van de Krol, N. Guijarro, F. Le Formal and K. Sivula, Chem. Mater., 2017, 29, 4952-4962.

130 Y. Hermans, A. Klein, H. P. Sarker, M. N. Huda, H. Junge, T. Toupance and W. Jaegermann, Adv. Funct. Mater., 2020, 30, 1910432.

131 A. K. Díaz-García, T. Lana-Villarreal and R. Gómez, J. Mater. Chem. A, 2015, 3, 19683-19687.

132 P. Yilmaz, D. Yeo, H. Chang, L. Loh and S. Dunn, Nanotechnology, 2016, 27, 345402 .
133 Y. Ren, F. Nan, L. You, Y. Zhou, Y. Wang, J. Wang, X. Su, M. Shen and L. Fang, Small, 2017, 13, 1603457.

134 M. I. Díez-García, V. Celorrio, L. Calvillo, D. Tiwari, R. Gómez and D. J. Fermín, Electrochim. Acta, 2017, 246, 365-371.

135 V. Celorrio, K. Bradley, O. J. Weber, S. R. Hall and D. J. Fermín, ChemElectroChem, 2014, 1, 1667-1671.

136 Q. Yu, X. Meng, T. Wang, P. Li, L. Liu, K. Chang, G. Liu and J. Ye, Chem. Commun., 2015, 51, 3630-3633.

137 M. I. Díez-García and R. Gómez, ChemSusChem, 2017, 10, 2457-2463.

138 G. P. Wheeler and K.-S. Choi, ACS Energy Lett., 2017, 2, 2378-2382.

139 B. Klahr, S. Gimenez, F. Fabregat-Santiago, T. Hamann and J. Bisquert, J. Am. Chem. Soc., 2012, 134, 4294-4302.

140 K. Shimizu and A. Lasia, Langmuir, 2012, 28, 7914-7920.

141 S. Gimenez, H. K. Dunn, P. Rodenas, F. Fabregat-Santiago, S. G. Miralles, E. M. Barea, R. Trevisan, A. Guerrero and J. Bisquert, J. Electroanal. Chem., 2012, 668, 119-125.

142 E. A. Ponomarev and L. M. Peter, J. Electroanal. Chem., 1995, 397, 45-52.

143 M. I. Díez-García and R. Gómez, ACS Appl. Mater. Interfaces, 2016, 8, 21387-21397.

144 W. H. Leng, Z. Zhang, J. Q. Zhang and C. N. Cao, J. Phys. Chem. B, 2005, 109, 15008-15023.

145 B. J. Ng, L. K. Putri, X. Y. Kong, Y. W. Teh, P. Pasbakhsh and S. P. Chai, Adv. Sci., 2020, 7, 1903171.

146 S. Chen, Y. Qi, C. Li, K. Domen and F. Zhang, Joule, 2018, 2, 2260-2288.

147 Y. Chen, X. Feng, Y. Liu, X. Guan, C. Burda and L. Guo, ACS Energy Lett., 2020, 5, 844-866.

148 M. S. Prévot and K. Sivula, J. Phys. Chem. C, 2013, 117, 17879-17893.

149 H. Döscher, J. F. Geisz, T. G. Deutsch and J. A. Turner, Energy Environ. Sci., 2014, 7, 2951-2956.

150 K. T. Fountaine, H. J. Lewerenz and H. A. Atwater, Nat. Commun., 2016, 7, 13706.

151 C. Lo Vecchio, S. Trocino, S. C. Zignani, V. Baglio, A. Carbone, M. I. D. Garcia, M. Contreras, R. Gómez and A. S. Aricò, Catalysts, $2020,10,525$.

152 A. Cots, P. Bonete, D. Sebastián, V. Baglio, A. S. Aricò and R. Gómez, ACS Appl. Mater. Interfaces, 2018, 10, 25393-25400. 